\title{
RANS and hybrid LES/RANS simulations of flow over a square cylinder
}

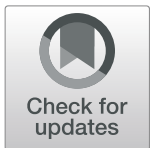

Jianghua Ke(1)

\author{
Correspondence: kejianghua@mail. \\ tsinghua.edu.cn \\ National Institute of Standards and \\ Technology, Gaithersburg, MD \\ 20899, USA
}

\begin{abstract}
Unsteady RANS (URANS), hybrid LES/RANS and IDDES simulations were conducted to numerically investigate the velocity field around, and pressures distribution and forces over a square cylinder immersed in a uniform, steady oncoming flow with Reynolds number $R e=21,400$. The vortex shedding responses in terms of Strouhal number, the pressure distribution, the velocity profile and the velocity fluctuations obtained by numerical simulations are compared with experimental data.

Compared with 2D URANS simulation, 3D simulations using hybrid LES/RANS and IDDES models provide more accurate prediction on the responses in the wake, including mean streamwise velocity profile and $r m s$ velocity fluctuations. This also results in more accurate prediction of time-averaged surface pressure coefficient on the rear surface obtained by 3D hybrid LES/RANS and IDDES simulations than by URANS simulation. When a hybrid LES/RANS model or IDDES model is used, a more accurate prediction for either pressure coefficient or velocity profile (especially in the far wake region) is not guaranteed by increasing the mesh resolution along the spanwise direction of the square cylinder.
\end{abstract}

Keywords: URANS, Hybrid LES/RANS, IDDES, Square cylinder, Turbulence modeling

\section{Introduction}

Three-dimensional high Reynolds number unsteady turbulent flows over bluff bodies (such as buildings and bridges) are of considerable importance in structural engineering applications. Massive separations are involved in this kind of flow; and periodic vortex shedding occurs at a well-defined frequency which also results in oscillatory lift and drag forces and renders the flow unsteady [1]. As a typical test case, turbulent flow over a square cylinder has been extensively studied both experimentally and numerically to investigate those features of bluff body flows. For example, Bearman [2], Nishimura and Taniike [3], and Noda et al. [4] measured the time-averaged surface pressure coefficient and rms pressure coefficient distributions on square cylinder at Reynolds numbers of 20,000, 10,000, and 68,900, respectively. Lyn et al. [5] performed experiments to measure the velocity fields as well as second order statistics of flow over a square cylinder at $\operatorname{Re}=21,400$. The numerical methods used to study the turbulent flow over square cylinder are basically URANS (Unsteady Reynolds-Averaged Navier-Stokes) or LES (Large Eddy Simulation). For example, Younis and Abrishamchi [6] investigated the

(c) The Author(s). 2019 Open Access This article is distributed under the terms of the Creative Commons Attribution 4.0 International License (http://creativecommons.org/licenses/by/4.0/), which permits unrestricted use, distribution, and reproduction in any medium, provided you give appropriate credit to the original author(s) and the source, provide a link to the Creative Commons license, and indicate if changes were made. 
three-dimensional vortex shedding from surface-mounted square cylinder with both LES and URANS. Studies by Younis and Przulj [7], Bouris and Bergeles [8], Lakehal et al. [9], Mahir [10]; Lim et al. [11], Srinivas et al. [12], Oka and Ishihara [13], and Bosch and Rodi [14] are also among the numerical investigations of flow over a square cylinder.

In recent years, URANS, LES and hybrid LES/RANS simulations have been employed to numerically investigate the unsteady wall-bounded turbulent flow at high Reynolds numbers in engineering applications. In a URANS simulation, an ensemble averaged version of the governing equations is solved, in which Reynolds stresses are introduced to represent the effects of turbulent fluctuations. LES is a technique in which the smallest scales of turbulence are modelled by a subgrid scale (SGS) model through a filtering operation, while the largest and most important scales are allowed to be resolved. In hybrid LES/RANS simulations, which have become increasingly popular in the past few years, RANS equations are solved in the inner layer whereas the filtered equations are solved in the outer layer of a boundary layer. Hybrid LES/RANS methods use a blending function to bridge the RANS and LES branches, which actually serves to merge the SGS and RANS eddy viscosities. There are various practices in the definition of the blending function, for instance, Gieseking et al. [15] find that the blending function is determined by the length scale ratio of the outer and inner layers. As an alternative to the hybrid LES/RANS method, Detached Eddy Simulation (DES), designed to address high Reynolds number separated flows, was first proposed by Spalart et al. [16]. In the DES method, a length scale is defined to determine which model will be used at a given grid point, with the results of RANS being implemented in attached boundary layers while the LES model is used in the outer layer and separation regions. Delayed Detached Eddy Simulation (DDES) and Improved Delayed Detached Eddy Simulation (IDDES) proposed by Spalart et al. [17] and Shur et al. [18], respectively, are updated versions of the DES model which address the problems of grid induced separation (GIS) and log-layer mismatch (LLM). Actually, the above mentioned DES method and its updated versions (DDES, IDDES) precede in time the Gieseking hybrid model discussed here, and the latter does not encounter the problems of GIS and LLM. The hybrid methodology has also been employed in the numerical studies on the turbulent flow over square cylinder by some investigators. For example, Roy et al. [19], Barone and Roy [20] conducted simulations of a low-speed square cylinder wake using DES method and compared the computational results of velocity and rms velocity fluctuations in the wake with the experiments; Morgan and Visbal [21] employed hybrid RANS/ILES methodology to simulate the same flow and compared the results with the experiments, Camarri et al. [22] developed a hybrid LES/RANS model which defines the eddy-viscosity by using a local blending of two eddy viscosities coming respectively from the RANS $k-\varepsilon$ closure and from the Smagorinsky subgrid-scale model, and they applied this model to the simulation of flow over the square cylinder and showed the distributions of eddy viscosity from both $k-\varepsilon$ and the Smagorinsky model. However, these investigations only provided limited computational results but didn't deliver the thorough data and flow properties for this kind of turbulent flow over a square cylinder, such as time-averaged pressure and drag distribution on each surface of 
square cylinder, the velocity and rms velocity profiles in the whole flow field, as well as the eddy viscosity distributions.

In this study, the performance of URANS (Menter's SST) model, hybrid LES/ RANS (Gieseking) model, as well as IDDES models with and without the Gieseking blending function (modified and original IDDES models) in predicting the characteristics of $3 \mathrm{D}$ turbulent flow over a square cylinder is investigated. The hybrid LES/RANS models (and IDDES model) have the merits of both URANS and LES in terms of computational costs and high fidelity [23]. Accurate solutions may not be obtained by URANS simulations because the chosen modeling approach may not model all the relevant physics of turbulent flow over square cylinder. For example, the mean streamwise velocity is overestimated while the streamwise normal stress is underestimated in the far wake region in the previous URANS simulations with $k-\varepsilon$ model by Younis [7]. Therefore, a hybrid LES/RANS method (aside from wall-resolved LES) may be a promising option for this case since only the near wall region is modeled. Based on how the blending function works, it is also worth to investigate the performance of different hybrid LES/ RANS models (in this study, Gieseking model, original IDDES model, modified IDDES model) in predicting the turbulent flow features when passing over a square cylinder. Besides, Ke [24] found that when Gieseking model is used for 3D simulations, mesh refinement along spanwise direction doesn't guarantee a solution that is in better agreement with the experimental measurements for turbulent flow at $\operatorname{Re}=1,000,000$ over a NACA 0012 airfoil under static stall, where the massive separation is involved as well. The effects of spanwise resolution of 3D mesh on the numerical solution of flow over a square cylinder by hybrid LES/RANS simulation are also investigated in this study.

In this work, the incompressible version of hybrid LES/RANS and URANS solver developed at North Carolina State University (NCSU) is used to investigate the aerodynamic characteristics of the flow over a square cylinder under the same Reynolds number $\operatorname{Re}=21,400$ as in the experiment performed by Lyn et al. [5]. The hybrid LES/RANS models and numerical methods used in this study are presented in section 2, including hybrid LES/RANS techniques previously developed at NCSU and the IDDES model. Section 3 discusses the 2D URANS, and 3D hybrid LES/RANS (and IDDES) computational results of flow over a square cylinder in comparison with the experimental measurements for velocity fields and second order statistics from Lyn et al. [5], and for pressure distribution from Bearman [2], Nishimura and Taniike [3] and Noda et al. [4]. Conclusions are drawn in section 4 .

\section{Hybrid LES/RANS models and numerical method}

The hybrid LES/RANS turbulence closure methods recently developed at NCSU are suitable for high Reynolds number, turbulent flows. The transition between a RANS component (used near solid surfaces) and the LES component (used in the outer parts of developing turbulent boundary layers and in free shear layers) is facilitated by the action of a flow-dependent blending function, which modifies the eddy viscosity field $\mu_{t}$ as follows: 


$$
\mu_{t}=\rho\left[(1-\Gamma) v_{t, s g s}+\Gamma \frac{k}{\omega}\right]
$$

where $\rho$ is density, $\Gamma$ is a time-dependent blending function that connects the RANS and LES branches. A model proposed by Lenormand et al. [25] is currently used for the subgrid-scale eddy viscosity $v_{t, s g s}$. Lenormand's model instead of Smagorinsky-like SGS models is what that hybrid LES/RANS model has always used. RANS eddy viscosity is determined by kinetic energy $k$ and modeled turbulence frequency $\omega$. The blending function $\Gamma$ is generally designed to transition the model from RANS to LES approximately as the boundary layer shifts from its logarithmic to its wake-like structure. As such, the RANS component acts as a wall-layer model for the majority of the flow, which is modeled as a large-eddy simulation. In the Gieseking model [15], a ratio of outer layer to the inner layer turbulent length scale is introduced into the argument for $\Gamma$

$$
\Gamma=\frac{1}{2}\left(1-\tanh \left[C_{s}\left(\frac{1}{\lambda_{N}^{2}}-1\right)\right]\right)
$$

where the constant $C_{s}=15.0$, and the turbulent length scale ratio $\lambda_{N}$ is determined by

$$
\begin{aligned}
& \lambda_{N} \equiv \frac{l_{\text {outer }}}{l_{\text {inner }}} \\
& l_{\text {outer }}=C_{N} \sqrt{\frac{10 v \bar{\omega}+\bar{k}+\bar{k}_{R}}{C_{\mu^{2}} \bar{\omega} \omega}} \quad l_{\text {inner }}=\kappa d
\end{aligned}
$$

In this expression, $C_{N}=1.5$ is a constant, $\bar{k}$ is the ensemble-averaged modeled turbulence kinetic energy, $\bar{k}_{R}$ is the ensemble-averaged resolved turbulence kinetic energy, $\omega$ and $\bar{\omega}$ are, respectively, instantaneous and ensemble-averaged modeled turbulence frequencies, $C_{\mu}=0.09, d$ is the distance to the nearest wall, and $\kappa$ is von Karman constant. Here all the ensemble-averaged variables are averaged over time.

The Improved Delayed Detached Eddy Simulation (IDDES) combines DDES with an improved hybrid RANS-LES model aimed at wall modelling in LES (WMLES), and switches between the two branches via a blending function. It is essentially a hybrid RANS-LES model that provides a flexible and convenient scale-resolving simulation for high Reynolds number flows. The SST-IDDES model used in this study is based on modifying the destruction term in the $k$-equation of the Menter's SST (Shear-Stress Transport) model [26]. (Note that the $\omega$-equation remains unchanged)

$$
\frac{\partial}{\partial t}(\rho k)+\frac{\partial}{\partial x_{j}}\left(\rho u_{j} k\right)=\tau_{i j} \frac{\partial u_{i}}{\partial x_{j}}-\beta^{*} \rho \omega k F_{I D D E S}+\frac{\partial}{\partial x_{j}}\left[\left(\mu+\sigma_{k 1} \mu_{t}\right) \frac{\partial k}{\partial x_{j}}\right]
$$

where $u$ is the velocity field, $\tau_{i j}$ is shear stress tensor, model constants $\beta^{\prime \prime}=0.09, \sigma_{k 1}$ $=0.85$, and $\mu$ is molecular viscosity. $F_{I D D E S}$ is a function based on the RANS turbulent length scale $l_{R A N S}$ and the LES length scale $l_{L E S}$ [18]

$$
F_{I D D E S}=\frac{l_{R A N S}}{l_{I D D E S}}, \quad l_{R A N S}=\frac{\sqrt{k}}{\beta^{*} \omega}
$$




$$
l_{I D D E S}=\tilde{f}_{d}\left(1+f_{e}\right) l_{R A N S}+\left(1-\tilde{f}_{d}\right) l_{L E S}
$$

where the blending function $\tilde{f}_{d}$ is defined by $\tilde{f}_{d}=\max \left\{\left(1-f_{d t}\right), f_{B}\right\}$, with $f_{d t}=1-$ $\tanh \left[\left(8 r_{d t}\right)^{3}\right]$. Here $r_{d t}$ is related to delaying function, $f_{B}$ is an empirical blending function; both were given in defining the length scale of WMLES branch by Shur et al. [18]. The elevating function $f_{e}$ is another empirical function [18].

In this study, both Gieseking's hybrid LES/RANS model and Spalart's original IDDES model were used for the 3D simulation of flow over a square cylinder. In addition, the original IDDES model was combined with the Gieseking model, which means eddy viscosity was determined by a hybrid method presented in Eq. (1) instead of just by Menter's SST model with the revised $k$-Eq. (5). The introduction of the Gieseking's blending function also reduces the production of turbulent kinetic energy $k$, and therefore increases the LES activity (and restrain RANS activity) in the simulation. It is usually favourable to have more LES content in hybrid LES/RANS simulations on a fine enough grid. Furthermore, since the role of subgrid scale model is not crucial, Lenormand subgrid scale model is used in the Gieseking model instead of the Smagorinsky-like subgrid model built in the original IDDES model. The original IDDES model combined with Gieseking's blending function is called the modified IDDES model here. Since Gieseking's model modifies the turbulence production while IDDES model modifies the turbulence destruction, the modified hybrid IDDES model modifies both. On the other hand, IDDES model can produce large eddy viscosities in free-shear regions, while the modified IDDES would not do this since Gieseking's model dominates in those regions.

Each hybrid LES/RANS (and IDDES) model is implemented into a finite volume Navier-Stokes solver that solves a low Mach number form of the Navier-Stokes equations. The artificial compressibility (AC) method is used to solve the PDE system of the incompressible flow by introducing a fake time derivative of pressure in the continuity equation [23]; and the primitive variable vector is used in the reconstruction. Inviscid fluxes are discretized using a variant of the piecewise parabolic method (PPM) [27] along with a low Mach number extension of Edwards's low-diffusion flux-splitting scheme [23, 28], whereas viscous and diffusive fluxes are discretized using second-order central differences. An implicit artificial compressibility method combined with sub-iteration procedure is employed to obtain second-order temporal accuracy.

\section{Computational results}

The experiment by Lyn et al. [5] was performed in a closed water channel. The side of the square cylinder was $D=40 \mathrm{~mm}$ and the incoming flow velocity (or free stream velocity) was $U=0.535 \mathrm{~m} / \mathrm{s}$, with $2 \%$ turbulence intensity level. Therefore, the incoming flow Reynolds number based on the side of the square cylinder is Re $=21,400$. In this work, both two-dimensional URANS and three-dimensional IDDES (and hybrid LES/RANS) simulations of the same flow configurations (except that incoming flow is smooth rather than turbulent) were performed to investigate flow characteristics, as well as the lift and drag forces.

Figure 1 shows the $x-y$ snapshot of the rectangular computational mesh used in this work, which extends $50 D$ and $20 D$ in $x$ - and $y$-direction, respectively. Since 

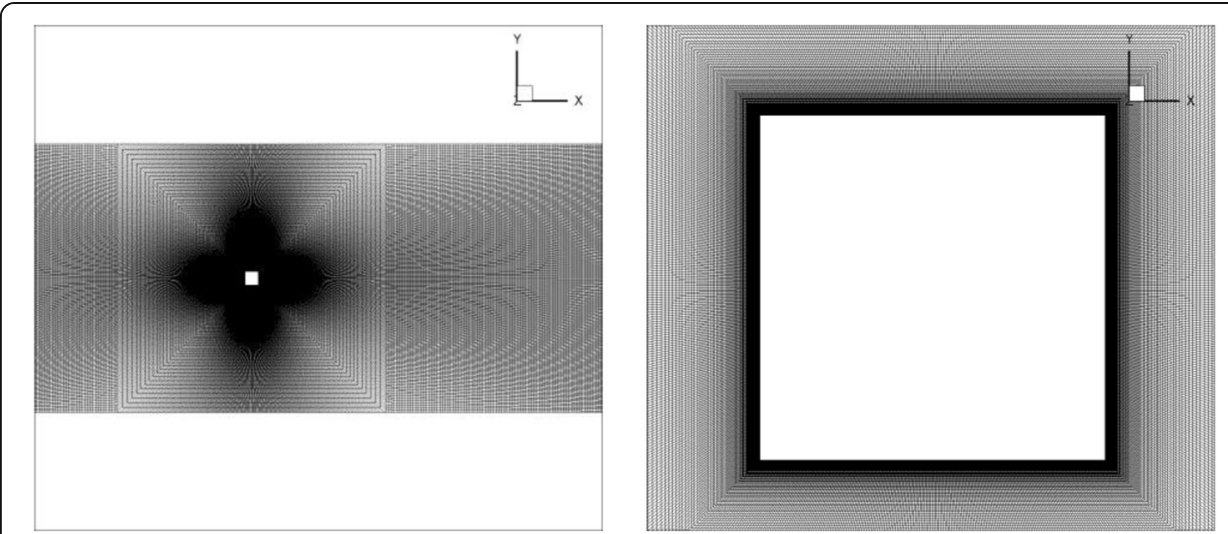

Fig. 1 Computational mesh: whole view (left); around square cylinder (right)

the $y^{+}$value is set less than 1 , no wall function is needed for either hybrid LES/ RANS or URANS simulations.

The experimental measurements of the Strouhal number (St), base-pressure coefficient $\left(-\mathrm{C}_{\mathrm{pb}}\right)$, time averaged drag coefficient $\left(\mathrm{C}_{\mathrm{D}}\right)$, rms lift $\left(\mathrm{C}_{\mathrm{L}}{ }^{\prime}\right)$ and $r m s$ drag $\left(\mathrm{C}_{\mathrm{D}}{ }^{\prime}\right)$ coefficients of flow over a square cylinder are summarized in Table 1. Since theoretically these aerodynamic characteristics are not affected by the incoming flow Reynolds number, they can be used for comparison with the simulation results in this work.

Table 1 Aerodynamic characteristics of flow over a square cylinder obtained by experiments

\begin{tabular}{|c|c|c|c|c|c|c|c|}
\hline & $\begin{array}{l}\text { Incoming flow } \\
\text { intensity (\%) }\end{array}$ & $\mathrm{Re} / 10^{3}$ & St & $-C_{p b}$ & $C_{D}$ & $C_{D}^{\prime}$ & $C_{L}^{\prime}$ \\
\hline Lyn and Rodi [5] & 2 & 21.4 & 0.132 & 1.6 & 2.1 & & \\
\hline Vickery [29] & smooth & 100 & 0.118 & 1.31 & & & 1.33 \\
\hline Noda and Nakayama [4] & 0.2 & 68.9 & 0.131 & 1.483 & 2.164 & 0.207 & 1.18 \\
\hline Noda and Nakayama [4] & 5.3 & 68.9 & 0.133 & 1.318 & 1.989 & 0.203 & 1.105 \\
\hline \multirow[t]{2}{*}{ Bearman and Obasaju [2] } & $<0.04$ & 11 to 47 & 0.127 & 1.65 & & & \\
\hline & $<0.04$ & 20 & & & & & 1.2 \\
\hline Nishimura and Taniike [3] & 0.1 & 40 & & 1.3 & 2.32 & 0.245 & 1.32 \\
\hline \multirow[t]{3}{*}{ Igarashi [30] } & 0.5 & 22 & $0.126 \sim 0.131^{a}$ & $1.29 \sim 1.35^{\mathrm{a}}$ & & & \\
\hline & 0.5 & 37 & & & 2.22 & & \\
\hline & 0.5 & 56 & & & 2.18 & & \\
\hline Durao et al. [31] & 6 & 14 & 0.133 & & & & \\
\hline \multirow[t]{2}{*}{ Lee [32] } & smooth & 176 & & 1.42 & 2.17 & & \\
\hline & 0.5 & 176 & 0.122 & 1.29 & 2.06 & & \\
\hline \multirow[t]{6}{*}{ Liu et al. [33] } & 0.7 & 4.06 & & & 2.18 & & \\
\hline & 0.7 & 9.55 & & & 2.107 & & \\
\hline & 0.7 & 18.1 & & & 2.201 & & \\
\hline & 0.7 & 32.2 & & & 2.09 & & \\
\hline & 0.7 & 45.8 & 0.135 & & 2.084 & & 1.1 \\
\hline & 0.7 & 58.2 & & & 2.02 & & \\
\hline Alam et al. [34] & 0.5 & 47 & 0.128 & & 2.15 & 0.27 & 1.18 \\
\hline
\end{tabular}

${ }^{a}$ Experimental measurements differ from each other with different choice of side dimension (D) of the square cylinder 


\subsection{Results by 2D URANS, 3D hybrid LES/RANS, and IDDES simulations}

Two-dimensional URANS simulations were performed on a $2 \mathrm{D}$ mesh with the number of cells on each side of the square being 100, and the mesh size is 70,000, as shown in Table 2. The RANS model used in this study is Menter's Shear-Stress Transport model.

Based on the 2D mesh, on which the 2D URANS simulation was performed reliably and efficiently (as indicated by the 2D URANS computational results in the following sections), three-dimensional meshes for hybrid LES/RANS and IDDES simulations were generated by extrusion to a span width $(\operatorname{span} / \mathrm{D}=0.6)$ over the spanwise $(Z-)$ direction with various spanwise cell spacings $\Delta \mathrm{z}$, as shown in Table 2 . Here $\mathrm{N}_{\mathrm{xy}}$ is the number of cells of the $2 \mathrm{D}$ mesh, $\mathrm{N}_{\mathrm{z}}$ represents number of cells over span, while $\mathrm{N}$ represents total number of mesh cells in Table 2. Three-dimensional simulations by Gieseking, original IDDES, modified IDDES models on Mesh-B were performed to investigate the effect of different $3 \mathrm{D}$ models. For all cases, simulation times are about 250 flow-through times (the flow-through time is defined as $D / U$ ) or 34 vortex shedding periods, and the last 20 periods are sampled for data processing since it takes about 100 flow-through times (or 14 periods) for the flow to reach a statistically stationary state. The time step was chosen as $1.0 \times 10^{-6} \mathrm{~s}$.

\subsubsection{Vortex shedding frequency, lift and drag forces}

As shown in Table 3, the vortex shedding frequency obtained by 2D URANS simulation is $\mathrm{St}=0.137$, about $3 \%$ higher compared with the largest experimental value. Table 3 also indicates that the time averaged base pressure $\left(-C_{p b}\right)$ and drag $\left(C_{D}\right)$ coefficients obtained by the URANS simulation are within the range of experimental measurement values. For the $2 \mathrm{D}$ case, both $-\mathrm{C}_{\mathrm{pb}}$ and $\mathrm{C}_{\mathrm{D}}$ were averaged over 20 vortex shedding periods (with around 3600 time points). 2D URANS predictions of $r m s$ lift $\left(\mathrm{C}_{\mathrm{L}}{ }^{\prime}\right)$ were overestimated, while URANS estimates of rms drag $\left(\mathrm{C}_{\mathrm{D}}{ }^{\prime}\right)$ coefficient are poor and too much underestimated, with the error being over $96 \%$ of the minimum experimental value. Generally, the computational results obtained by URANS model on the specified $2 \mathrm{D}$ mesh are in good agreement with the experimental measurements except for $\mathrm{C}_{\mathrm{D}}$ '.

Table 3 also shows the aerodynamic characteristics calculated based on the solution of 3D hybrid LES/RANS and IDDES simulations on Mesh-B. Vortex shedding frequency in terms of Strouhal number (St) predicted by all 3D models on Mesh-B is within the range of experimental values, with the St predictions by Gieseking and modified IDDES models being close to each other and greater than the prediction obtained by original IDDES model. Time averaged base-pressure coefficients $\left(-\mathrm{C}_{\mathrm{pb}}\right)$ predicted by Gieseking and modified IDDES models on Mesh-B are also

Table 2 Characteristics of $2 \mathrm{D}$ and 3D meshes

\begin{tabular}{lllllll}
\hline & Span/D & $N_{x y}$ & $N_{z}$ & $N$ & $\Delta z(m)$ & $\Delta z / D$ \\
\hline 2D Mesh & 0.025 & 70,000 & 1 & 70,000 & 0.01 & 0.025 \\
3D Mesh-A & 0.6 & 70,000 & 48 & $3,360,000$ & 0.005 & 0.0125 \\
3D Mesh-B & 0.6 & 70,000 & 24 & $1,680,000$ & 0.01 & 0.025 \\
3D Mesh-C & 0.6 & 70,000 & 12 & 840,000 & 0.02 & 0.05 \\
3D Mesh-D & 0.6 & 70,000 & 6 & 420,000 & 0.04 & 0.1 \\
\hline
\end{tabular}


Table 3 Results obtained by URANS simulation on 2D mesh and 3D simulations on Mesh-B

\begin{tabular}{lllllll}
\hline Model & Mesh & St & $-C_{\mathrm{pb}}$ & $C_{\mathrm{D}}$ & $C_{\mathrm{L}^{\prime}}$ & $C_{\mathrm{D}^{\prime}}$ \\
\hline URANS & 2D Mesh & 0.137 & 1.35 & 2.07 & 1.37 & 0.06 \\
Gieseking & 3D Mesh-B & 0.127 & 1.28 & 2.02 & 1.24 & 0.180 \\
original IDDES & 3D Mesh-B & 0.122 & 1.4 & 2.13 & 1.27 & 0.272 \\
modified IDDES & 3D Mesh-B & 0.128 & 1.28 & 2.02 & 1.18 & 0.189 \\
Experiments & & $0.122 \sim 0.133$ & $1.3 \sim 1.6$ & $2.02 \sim 2.22$ & $1.18 \sim 1.32$ & $0.207 \sim 0.245$ \\
\hline
\end{tabular}

very close to each other and slightly below the experimental measurements, while the $-C_{\mathrm{pb}}$ prediction by original IDDES model on Mesh-B is more accurate compared with the experiments. Time averaged drag coefficients $\left(C_{D}\right)$ predicted by Gieseking and modified IDDES model are close to each other and smaller the $C_{D}$ prediction obtained by original IDDES model, all being within the range of experimental values. For all the $3 \mathrm{D}$ cases, both $-\mathrm{C}_{\mathrm{pb}}$ and $\mathrm{C}_{\mathrm{D}}$ were averaged over 20 vortex shedding periods (with about 3800 time points). The rms $\mathrm{C}_{\mathrm{L}}\left(\mathrm{C}_{\mathrm{L}}{ }^{\prime}\right)$ predictions by all 3D IDDES simulations on Mesh-B are in good agreement with the experiments, with the result by original IDDES model being greater than those predicted by Gieseking and modified IDDES models. The $r m s C_{D}\left(C_{D}^{\prime}\right)$ predictions by Gieseking and modified IDDES model on Mesh-B are close to each other and slightly underestimated, with the error being less than $13 \%$ of the minimum experimental value, while the $C_{D}$ ' prediction by original IDDES model on Mesh-B is slightly overestimated, with the error being $11 \%$ of the maximum experimental value. All the $3 \mathrm{D}$ models provide more accurate predictions on $\mathrm{C}_{\mathrm{L}}{ }^{\prime}$ and $\mathrm{C}_{\mathrm{D}}{ }^{\prime}$ than the $2 \mathrm{D}$ URANS model compared with the experimental measurements. Basically, the values of most aerodynamic characteristics predicted by Gieseking model and the modified IDDES model are close to each other, but slightly different from those predicted by the original IDDES model.

\subsubsection{Surface pressure coefficient distribution}

Figure 2 shows comparisons of the time-averaged surface pressure coefficient $\left(\mathrm{C}_{\mathrm{p}}\right)$ and rms pressure coefficient $\left(\mathrm{C}_{\mathrm{p}}{ }^{\prime}\right)$ distributions obtained by $2 \mathrm{D}$ and 3D simulations with experimental data from Noda et al. [4], Nishimura and Taniike [3] and Bearman [2]. The average $C_{p}$ and $C_{p}$ ' predictions of $2 D$ URANS simulation are generally in good agreement with the experiments. The $C_{p}$ and $C_{p}{ }^{\prime}$ distributions obtained by 3D hybrid LES/ RANS and IDDES simulations on Mesh-B are also shown on Fig. 2. The average surface pressure coefficient distributions predicted by Gieseking and modified IDDES models are close to each other on all the four surfaces of the square cylinder. The predictions of average $C_{p}$ distribution on the front surface by all the 3D models are close to each other and in good agreement with the experiments, the predictions of average $\mathrm{C}_{\mathrm{p}}$ distribution on the top surface by all the 3D models are also close to each other but slightly overestimated compared with the experiments. The predictions of average $C_{p}$ distribution on the rear surface (towards the wake) are slightly overestimated by all the $3 \mathrm{D}$ models, with the prediction by original IDDES model being the most accurate compared with the experiments, and the predictions of average $C_{p}$ distribution on the bottom 


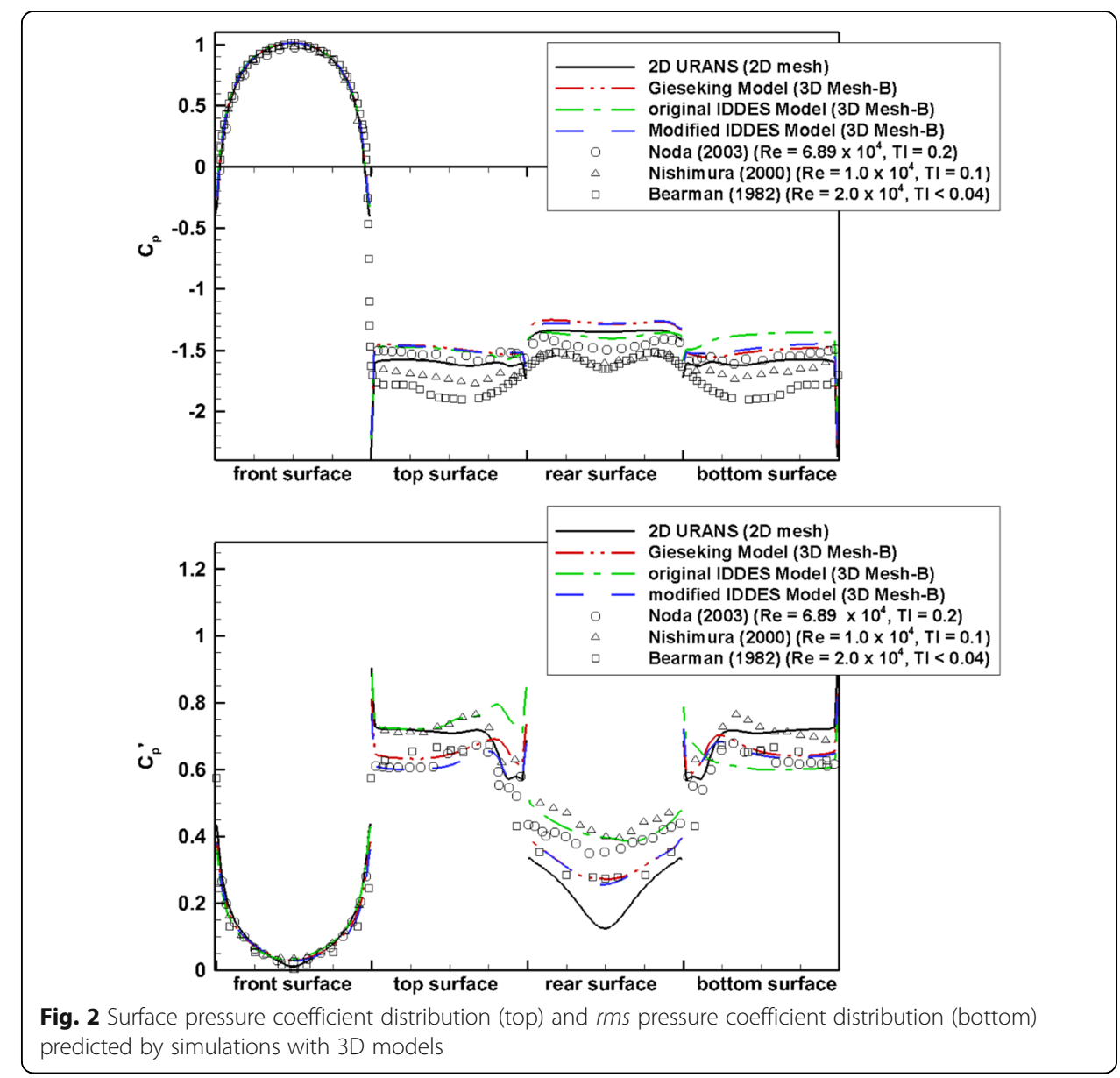

surface are also slightly overestimated by all the 3D models, with the prediction by original IDDES model being the least accurate compared with the experiments. The 3D hybrid LES/RANS and IDDES simulations on Mesh-B do not show better performance than the URANS simulation on $2 \mathrm{D}$ mesh in the prediction of averaged $\mathrm{C}_{\mathrm{p}}$ distribution over the top, rear and bottom surfaces of the square cylinder compared with the experiments. In the prediction of rms pressure coefficient $\left(\mathrm{C}_{\mathrm{p}}{ }^{\prime}\right)$, the results obtained by Gieseking and modified IDDES models on Mesh-B are close to each other on all surfaces. The predictions of $\mathrm{C}_{\mathrm{p}}$ ' by all $3 \mathrm{D}$ simulations are more accurate than that obtained by 2D URANS simulation on the rear surface, with the $C_{p}$ ' prediction by original IDDES simulation being in the best agreement with the experiments by both Noda et al. [4] and Nishimura and Taniike [3], while the $C_{p}$ ' prediction by Gieseking and modified IDDES simulations being in the best agreement with the experiments by Bearman [2]. As shown on Fig. 2, near the center of rear surface the $C_{p}$ predictions differ from the experimental data by $10 \%$ at most, whereas the $\mathrm{C}_{\mathrm{p}}{ }^{\prime}$ predictions are underestimated by as much as $75 \%$. $2 \mathrm{D}$ URANS simulation significantly underestimates the rms pressure coefficient on the rear surface because the solution in the wake region obtained by URANS model is not as accurate as IDDES model. It is also noticed that the $C_{p}$ and $C_{p}{ }^{\prime}$ results obtained by $3 D$ hybrid 
methods are not symmetrical on the top and bottom surfaces of the square cylinder, this is because sample points of only 20 vortex shedding periods were averaged over time.

\subsubsection{Velocity profiles and rms velocity fluctuations}

The error bands of Lyn's experimental data shown in Figs. 3, 4, 5, 6 and 7 were calculated by multiplying their maximum measurement values by the uncertainty of the measurement $( \pm 5 \%)$ quoted by Lyn et al. [5].

Figure 3 shows the profiles of mean streamwise and cross-stream velocity components ( $u$ and $v$ ) upwind of the wake region predicted by 2D and 3D simulations. The prediction of mean streamwise velocity profile (top plot of Fig. 3) obtained by URANS simulation on 2D mesh is in good agreement with Lyn's
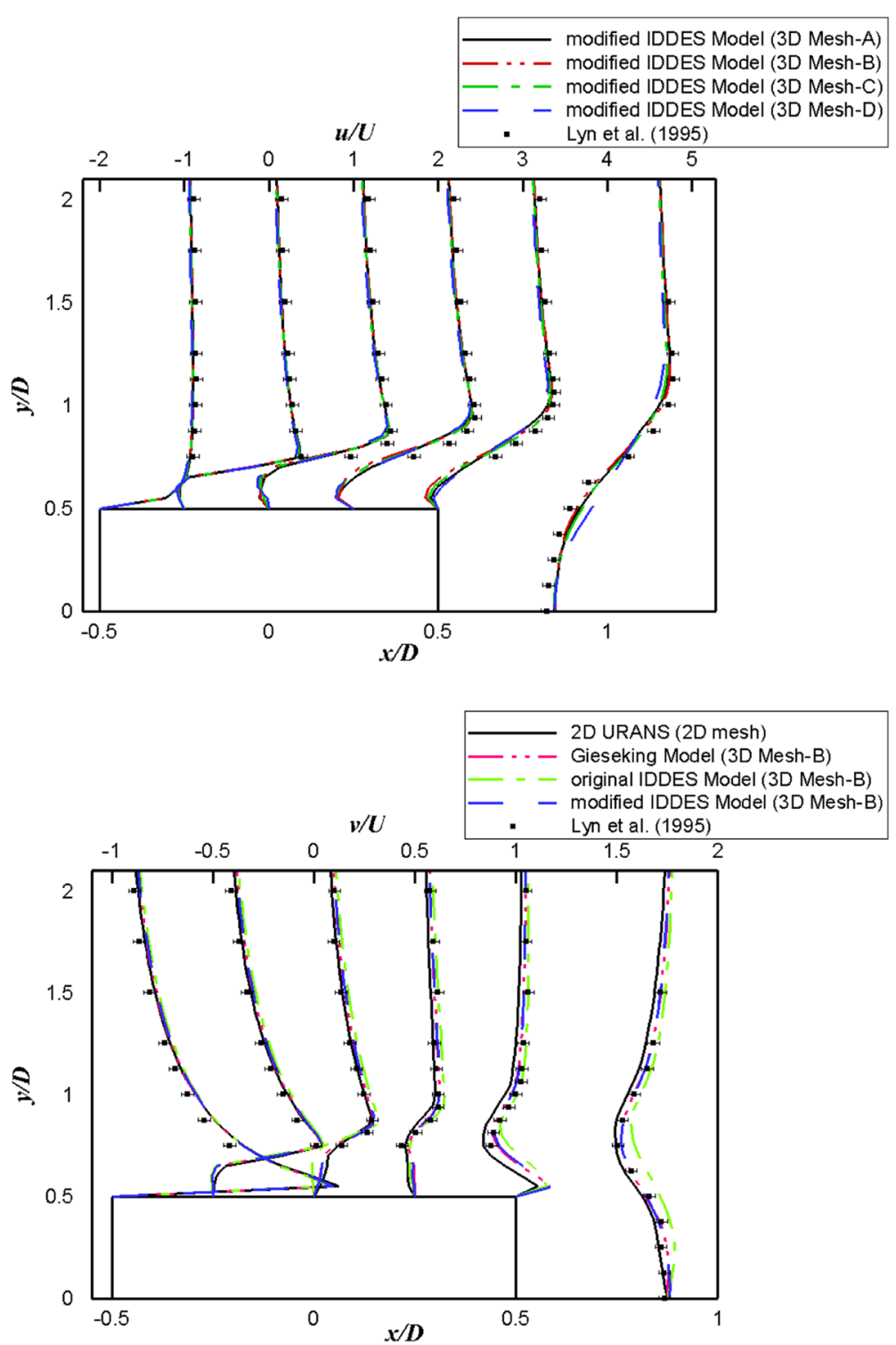

Fig. 3 Mean streamwise (top) and cross-stream (bottom) velocity profiles at different locations near the cylinder predicted by simulations with URANS model and 3D models 


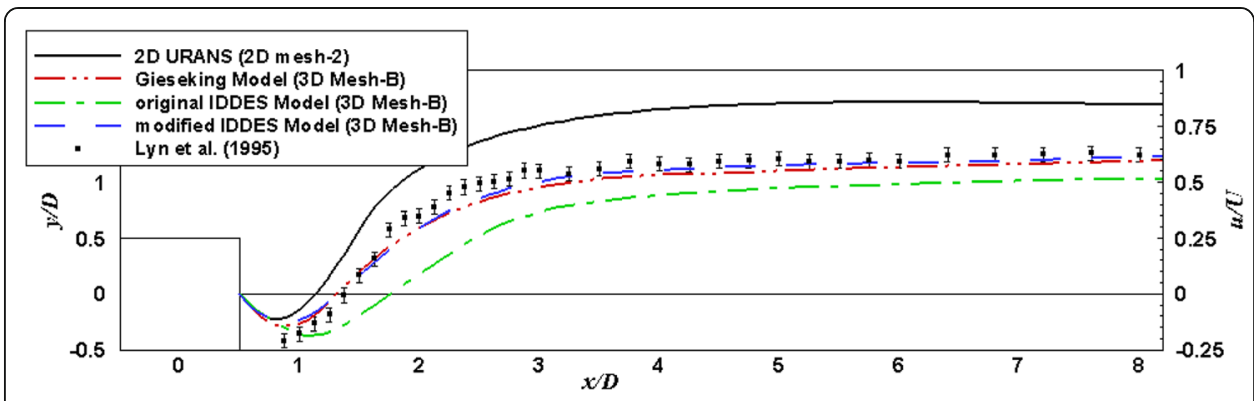

Fig. 4 Mean streamwise velocity profile along the wake centerline predicted by simulations with URANS model and 3D models

experimental data upwind of the wake region, and the prediction of mean cross-stream velocity profile (bottom plot of Fig. 3) obtained by 2D URANS simulation is in good agreement with Lyn's experimental data in the upwind region. In the downstream region $(x / D>0.25)$, the prediction of mean cross-stream velocity is slightly underestimated compared with Lyn's data, with the maximum relative error being over 5\%. Figure 3 also shows the profiles of mean streamwise and cross-stream velocity components $(u$ and $v)$ upwind of the wake and near wake $(x /$ $D=0.875)$ predicted by 3D hybrid LES/RANS and IDDES simulations on Mesh-B. Both the $u$ and $v$ velocity profiles predicted by Gieseking and modified IDDES models are close to each other. In the predictions of mean $u$ profile, all 3D models obtained accurate results against Lyn's measurements in the region above the shear layer. However, the mean $u$ was underestimated in the region below shear layer (at $x / D=0,0.25$, and 0.5 ) by all $3 \mathrm{D}$ models, with prediction by original IDDES model being the least accurate (up to 30\% relative error) compared with Lyn's experimental measurements, while the URANS simulation on 2D mesh obtained more accurate prediction in this region. In the predictions of mean $v$ profile, results obtained by both 3D models and 2D URANS model are slightly overestimated in the upstream region $(x / D \leq 0)$ compared with Lyn's measurements, with the prediction obtained by original IDDES model being the least accurate, while the prediction by 2D URANS simulation being the most accurate. In the downstream region $(x / D>0)$ and near wake region $(x / D=0.875)$, mean $v$ profile predictions by Gieseking and modified IDDES models match the Lyn's data well, while prediction by original IDDES model slightly overestimates the value with the maximum relative error over $5 \% .2$ D URANS simulation slightly underestimates the mean $v$ velocity in the downstream and near wake regions, with the maximum relative error greater than $5 \%$. Generally, Gieseking and the modified IDDES model perform the best in the prediction of mean $u$ and $v$ profiles among all the 3D and 2D models.

As shown in Fig. 4, the mean streamwise velocity profile by 2D URANS simulation is overestimated along the wake centerline, while the predictions of mean streamwise velocity $(u)$ profile along the wake centerline by 3D models are much closer to Lyn's experiments than that of URANS simulation, as shown in Fig. 4. Among all the 3D simulations, the modified IDDES model provides the best performance in predicting the mean streamwise velocity against the experiments, especially along the centerline in the far wake region $(x / D>2.0)$, while the original IDDES model provides the least 

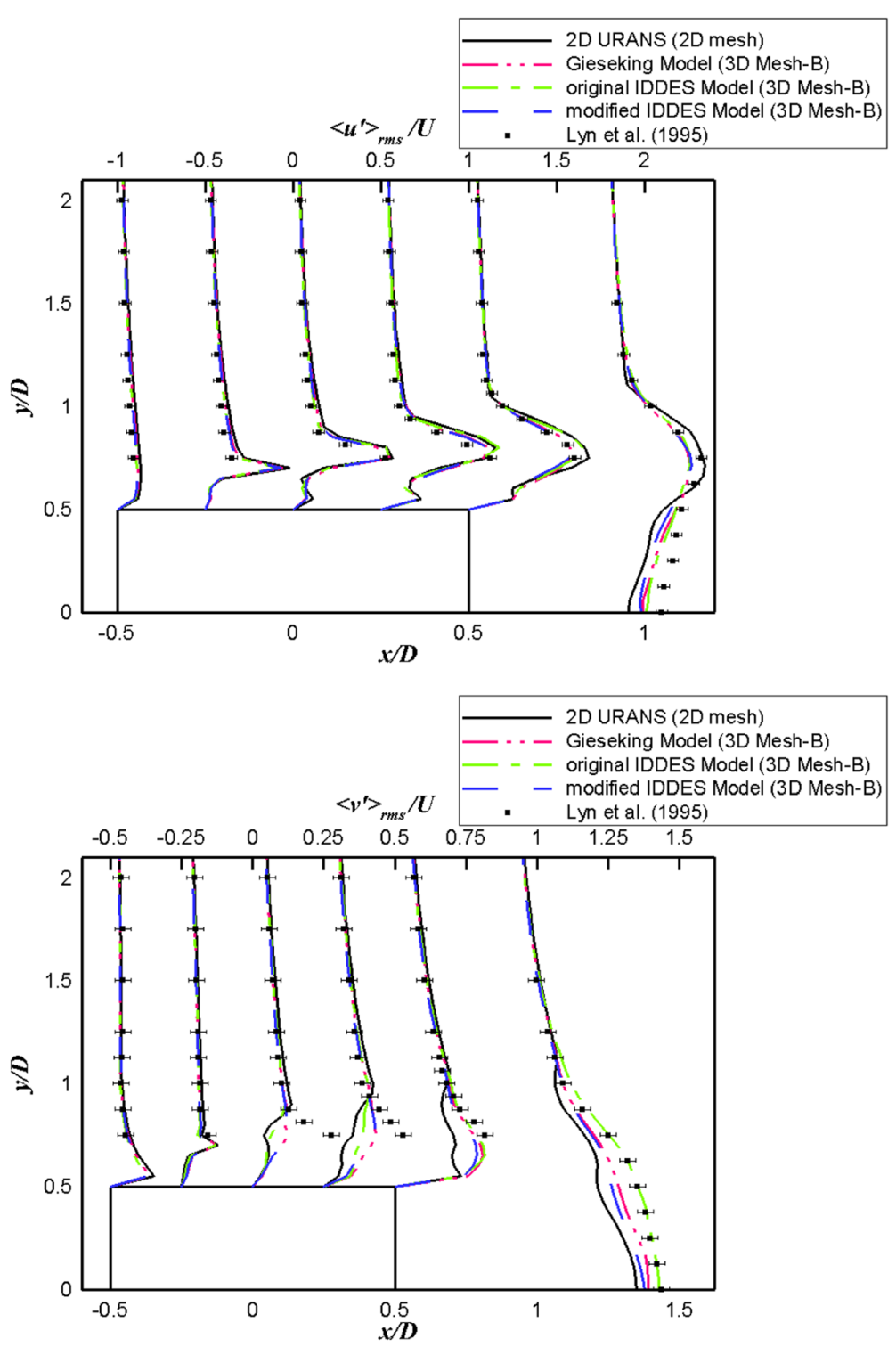

Fig. 5 rms streamwise (top) and cross-stream (bottom) velocity fluctuations at different locations before wake predicted by simulations with URANS model and 3D models

accurate prediction by underestimating the mean $u$ profile along the centerline in the region of $x / D>1.0$.

The rms velocity fluctuations and Reynolds-averaged shear stress were also calculated based on the solution of 2D and 3D simulations compared with Lyn's experimental data. Figure 5 shows the rms streamwise $\left(\langle u\rangle_{r m s} / U=\sqrt{\overline{u^{\prime} u^{\prime}}} / U\right)$ and cross-stream $\left.(<v\rangle_{r m s} / U=\sqrt{\overline{v^{\prime} v^{\prime}}} / U\right)$ velocity fluctuations upwind of the wake and in the near wake region. Prediction of $r m s$ streamwise velocity fluctuation upwind of wake region by $2 \mathrm{D}$ URANS simulation is in good agreement with Lyn's experiments, but is underestimated in the near wake, with relative error up to $50 \%$. URANS prediction of rms cross-stream velocity fluctuation in the region farther away from the square cylinder wall is also in 


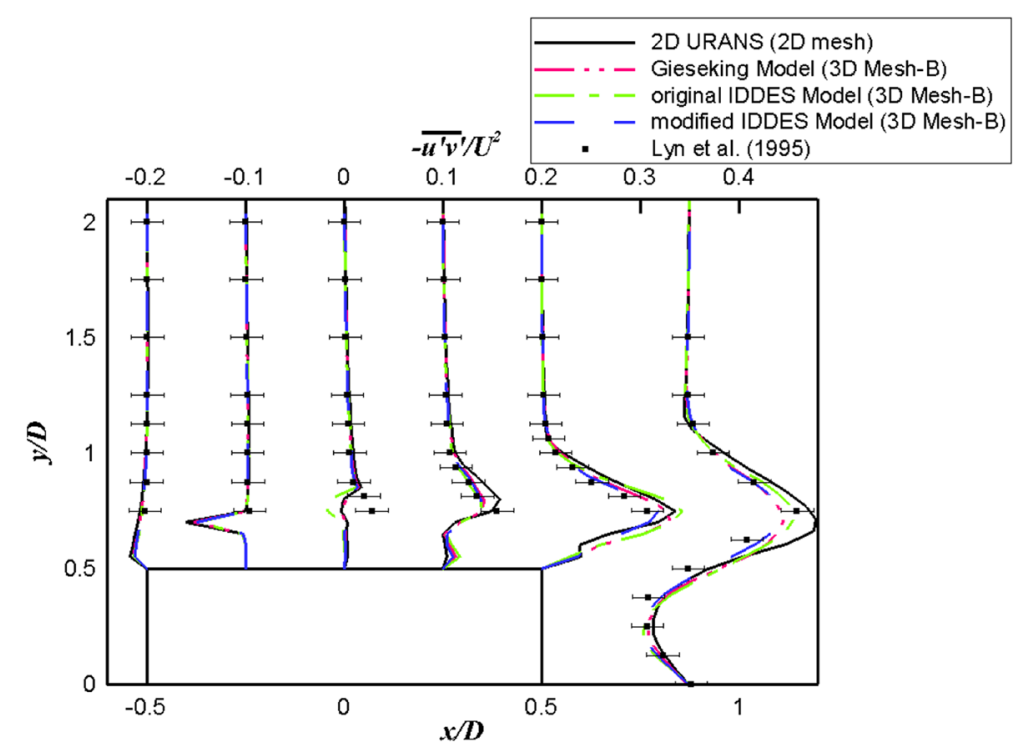

Fig. 6 Reynolds-averaged shear stress profile at different locations before wake predicted by simulations with URANS model and 3D models

good agreement with the experiment. However, it is poor in the region close to the cylinder wall and in near wake region: the maximum relative errors reach up to $80 \%$ and $70 \%$ at some positions close to cylinder along $x / D=0$ and 0.25 , respectively, while the relative error is up to $25 \%$ near the wake. Figure 5 also indicates that both the $\left\langle u^{\prime}\right\rangle_{\text {rms }} l$ $U$ and $\left\langle v^{\prime}\right\rangle_{r m s} / U$ velocity fluctuation profiles predicted by Gieseking and modified IDDES models are close to each other. In the predictions of mean $\left\langle u^{\prime}\right\rangle_{r m s} / U$ profile, results obtained by all 3D models are in good agreement with Lyn's measurements in the upstream region $(x / D \leq 0)$, being more accurate than the results obtained by $2 \mathrm{D}$ URANS simulation which slightly overestimates the values compared with the Lyn's data. In the region below downstream $(x / D>0)$ shear layer, $\left\langle u^{\prime}\right\rangle_{r m s} / U$ profile predictions by Gieseking and modified IDDES model also match the Lyn's data well, while prediction by original IDDES model underestimates the value with maximum relative error up to $6 \%$. 2D URANS simulation also underestimates $\left\langle u^{\prime}\right\rangle_{r m s} / U$ within that region, with maximum relative error greater than $6 \%$. In the near wake $(x / D=0.875)$, especially near centerline, $\left\langle u^{\prime}\right\rangle_{r m s} / U$ was underestimated by both $3 \mathrm{D}$ models and $2 \mathrm{D}$ URANS model, with results obtained by URANS model being the least accurate compared with the Lyn's data. In the predictions of mean $\left\langle v^{\prime}\right\rangle_{r m s} / U$ profile, results obtained by $3 \mathrm{D}$ models are in good agreement with Lyn's measurements in the upstream region $(x / D \leq 0)$ (except the below shear layer region at $x / D=0.0$ and 0.25 ), being more accurate than the results obtained by 2D URANS simulation which slightly overestimates the values in the region above shear layer and significantly underestimates the values below shear layer. In the region below shear layer at $x / D=0.0$ and $0.25,\left\langle v^{\prime}\right\rangle_{r m s} / U$ is also underestimated by 3D models, with results by original IDDES model being less accurate than those obtained by Gieseking and modified IDDES model compared with the Lyn's measurements. In the near wake region $(x / D=0.875)$, prediction of $\left\langle v^{\prime}\right\rangle_{\text {rms }} / U$ by original IDDES model is in the best agreement with the Lyn's data and Gieseking and modified 


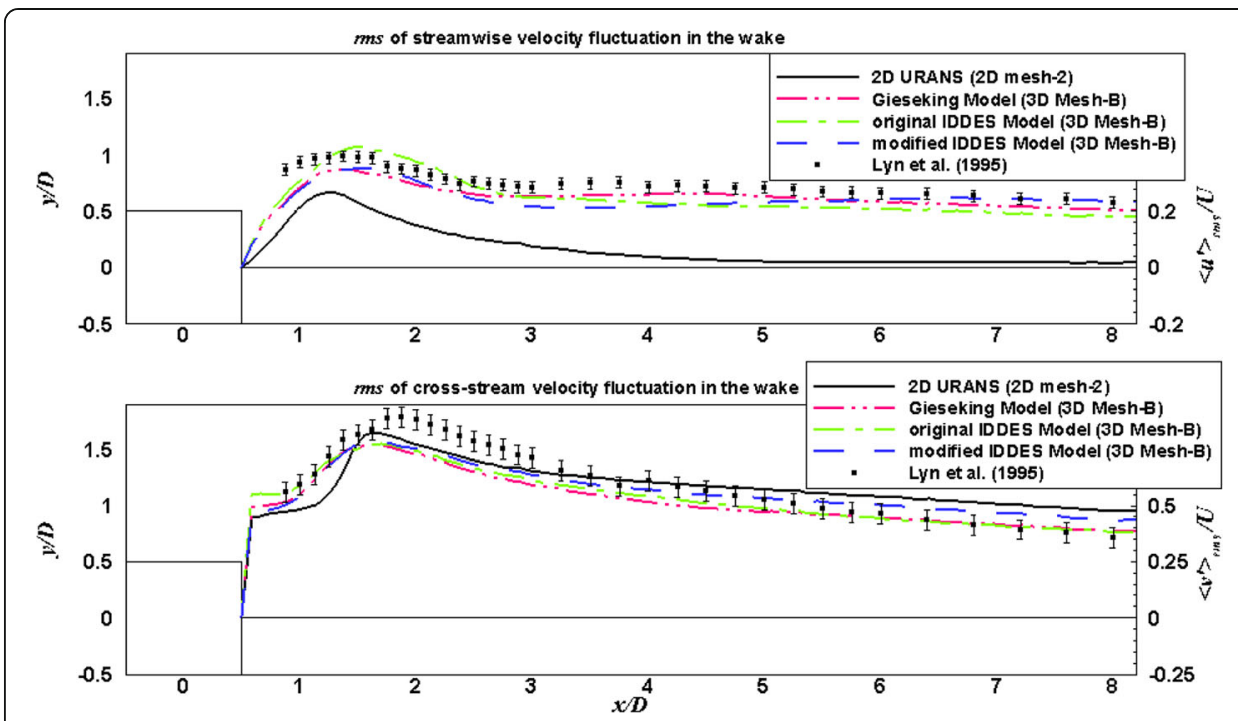

Fig. 7 rms streamwise (top) and cross-stream (bottom) velocity fluctuations along the wake centerline predicted by simulations with URANS model and 3D models

IDDES models slightly underestimated the values with maximum error up to $10 \%$, while the prediction by 2D URANS model is the least accurate against the Lyn's data. These indicate that the 3D models perform better in predicting $\left\langle u^{\prime}\right\rangle_{r m s} / U$ and $\left\langle v^{\prime}\right\rangle_{r m s} / U$ upwind of and near the wake than the 2D URANS model.

Figure 6 indicates that the prediction of Reynolds-averaged shear stress $\left(-\overline{u^{\prime} v^{\prime}} / U^{2}\right)$ profile upwind of the wake and in the near wake region by 2D URANS simulation is in good agreement with the experiments, with the maximum relative error being less than $10 \%$ with respect to the reported experimental data. The Reynolds-averaged shear stress $\left(-\overline{u^{\prime} v^{\prime}} / U^{2}\right)$ prediction by 3D hybrid LES/RANS and IDDES simulations are comparable to that obtained by 2D URANS simulation in the region above shear layer, all being in good agreement with the experimental measurements, as shown in Fig. 6. In the region below shear layer at $x / D=0.25$ and 0.5 , Gieseking and modified IDDES models provide the most accurate prediction on $-\overline{u^{\prime} v^{\prime}} / U^{2}$ against the Lyn's data, while original IDDES and 2D URANS models slightly overestimated the values with maximum error up to $10 \%$ and $8 \%$, respectively. In the near wake region $(x / D=0.875)$, $-\overline{u^{\prime} v^{\prime}} / U^{2}$ predictions by all the 3D models are close to each other and in good agreement with the Lyn's data, being more accurate than those obtained by 2D URANS model which overestimates the values with maximum error up to $10 \%$.

Figure 7 shows the profiles of rms streamwise velocity (top) and cross-stream velocity (bottom) fluctuations in the wake obtained by URANS simulation on 2D mesh and hybrid LES/RANS and IDDES simulations on 3D Mesh-B. The 2D URANS prediction of rms streamwise velocity fluctuation is underestimated (with maximum relative error up to $95 \%$ ), while $r m s$ cross-stream velocity fluctuation is underestimated in the near wake (with maximum error up to $25 \%$ ) and overestimated in the far wake (with maximum relative error up to $30 \%$ ). Basically, compared with the Lyn's experimental measurements, the URANS model produced good predictions in terms of streamwise and cross-stream velocity profiles, rms 
streamwise and cross-stream velocity fluctuations, as well as Reynolds shear stress upwind of the wake. However, the URANS predictions are more erroneous in the wake and close to the cylinder. Figure 7 also shows the profiles of rms streamwise velocity (top) and cross-stream velocity (bottom) fluctuations along the centerline in the wake region obtained by 3D hybrid LES/RANS and IDDES simulations. In the predictions of $\left\langle u^{\prime}\right\rangle_{r m s} / U$ along the centerline, although the 3D models slightly underestimate the values in most of the wake region, the predictions are much more accurate than those obtained by 2D URANS simulation against the Lyn's data. In the rms streamwise velocity fluctuation predictions, the original IDDES model performs better than Gieseking and modified IDDES models in the near wake $(x / D<3.0)$ while the modified IDDES model performs the best in the far wake region $(x / D>5.5)$. In the predictions of $\left\langle v^{\prime}\right\rangle_{r m s} / U$ along the centerline, the original IDDES model obtains the most accurate results in the near wake region $(0.5<x / D<1.5)$ compared with the experiments, while Gieseking and modified IDDES models obtain slightly underestimated values with maximum error less than $5 \%$. In the same region, 2D RANS simulation obtains the least accurate prediction by underestimating the values with maximum error up to $15 \%$ against the Lyn's data. In the far wake region $(x / D>4.0)$, the $r m s$ cross-stream velocity fluctuations predicted by the original IDDES and Gieseking models are in good agreement the Lyn's data, while the modified IDDES model and 2D URANS model overestimate the value, with maximum error being up to $7 \%$ and $12 \%$, respectively.

Generally, the 3D hybrid LES/RANS and IDDES simulations show better performance than 2D URANS simulations in the predictions of surface pressure coefficient and rms pressure coefficient, velocity profiles and second order statistics (rms velocity fluctuations), especially in the wake region. Figure 8 shows the iso-surface of vorticity based on the URANS solution on 2D mesh (left) and the 3D solution by the modified IDDES model on Mesh-B (right). The 3D solution by the modified IDDES model captures the 3D turbulent structures of the unsteady flow, especially in the far wake region, while 2D URANS solution fails to. That is the reason why 3D models provide more accurate predictions on streamwise velocity profile and $r m s$ velocity fluctuations in the wake region than 2D URANS model does. Figure 9 shows the contours of the time-averaged streamwise velocity based on the solutions of URANS simulation on 2D

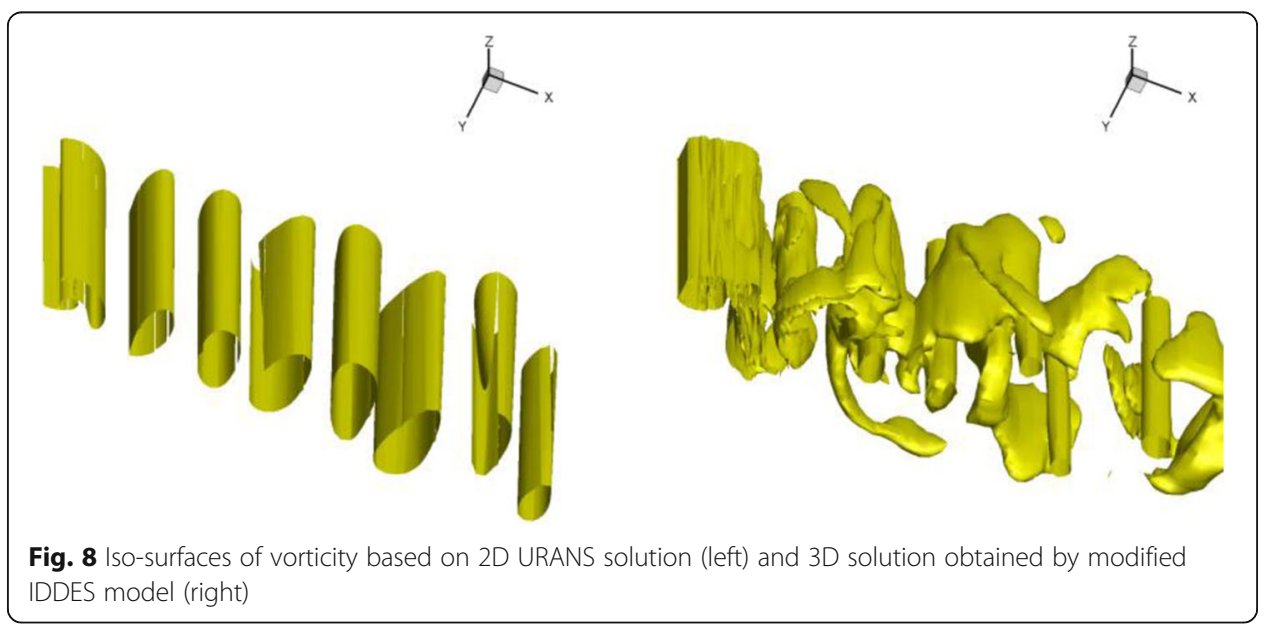




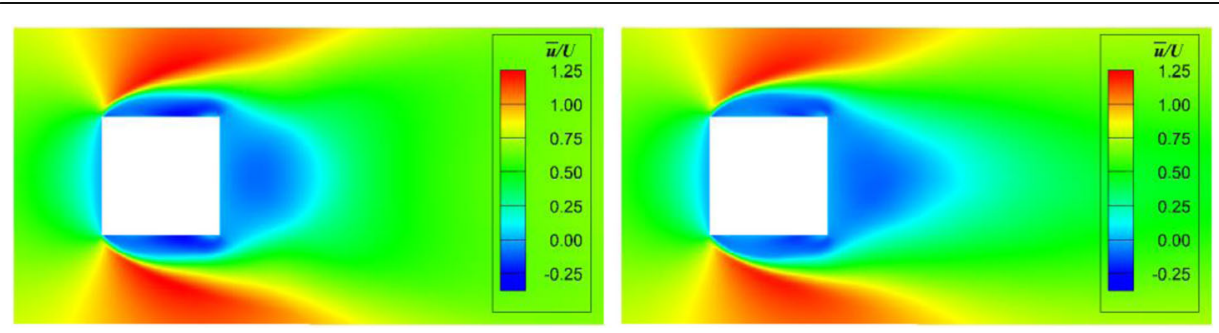

Fig. 9 Streamwise velocity contours based on 2D URANS solution (left) and 3D solution obtained by modified IDDES model (right)

mesh and 3D simulation by the modified IDDES model on Mesh-B, indicating both URANS and modified IDDES model correctly predict that the separation starts from the corner of the square cylinder. However, Fig. 9 also indicates that the separation region predicted by the modified IDDES simulation extends longer towards the wake compared with that predicted by the URANS simulation. Again, this is due to the fact that the modified IDDES model resolves the 3D turbulent structures in the wake while the URANS model doesn't. Other 3D (Gieseking and the original IDDES) models perform similarly as the modified IDDES model in resolving the 3D turbulent structures in the wake region.

Among all 3D simulations on Mesh-B, the predictions obtained by Gieseking and the modified IDDES models are very similar in terms of the Strouhal number, base-pressure coefficient, lift coefficient, rms lift and drag coefficients, surface pressure coefficient distribution, rms pressure coefficient distribution, $u$ and $v$ velocity profiles, and $u$ and $v$ velocity fluctuation profiles. The predictions obtained by the original IDDES model slightly vary from those by Gieseking and the modified IDDES models in most cases. Figure 10 shows the contours of time-averaged eddy viscosity (normalized by molecular viscosity) obtained by simulations using URANS (Menter's SST) model on 2D mesh and different 3D models on Mesh-B. The time-averaged eddy viscosity obtained by 2 D URANS simulation is very large in the separation and wake regions since
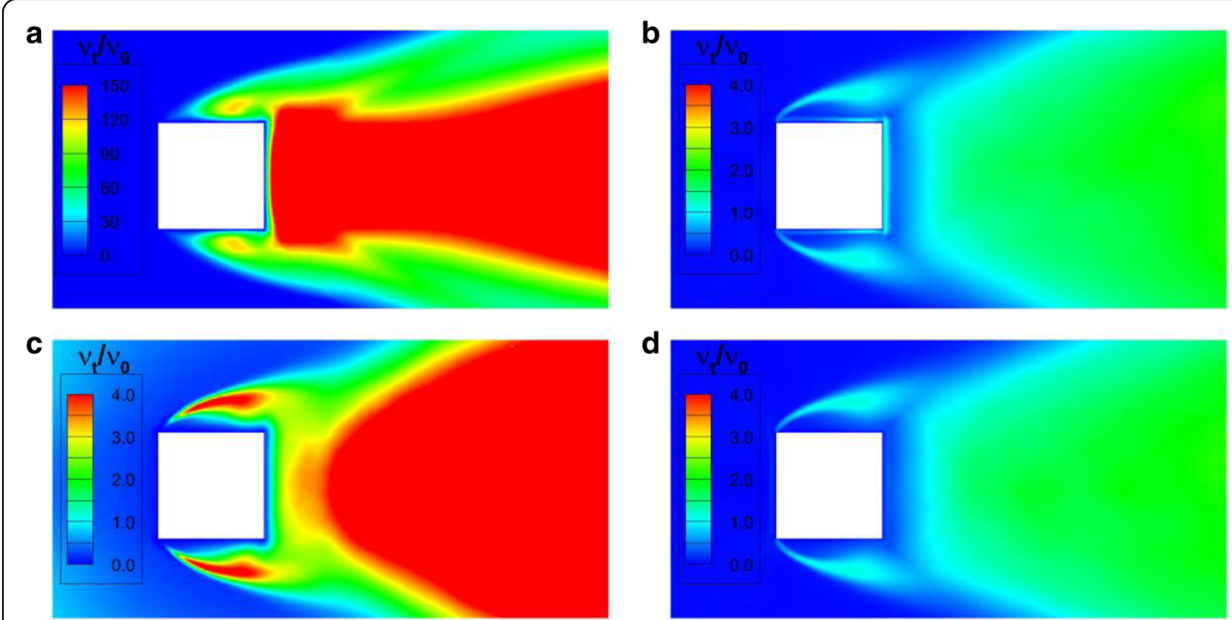

Fig. 10 Time-averaged eddy viscosity contours based on a 2D simulation by URANS model on 2D mesh; $\mathbf{b}$ 3D simulation by Gieseking model on Mesh-B; $\mathbf{c}$ 3D simulation by the original IDDES model on Mesh-B; $\mathbf{d}$ 3D simulation by the modified IDDES model on Mesh-B 
all the turbulent behaviours are modelled. The contours of time-averaged eddy viscosity obtained by Gieseking and the modified IDDES models look similar except that Gieseking model results in slightly larger values of time-averaged eddy viscosity close to the cylinder wall. This explains the similarity of the predictions obtained by the two models. The original IDDES model results in near zero time-averaged eddy viscosity close to the wall and relatively lager values of eddy viscosity in the rest of the separation and wake regions compared with those obtained by Gieseking and the modified IDDES models, which means more RANS activity and less LES activity in those regions. In the fully separated and wake regions, the dominant and most turbulent vortices are large enough to be resolved by the 3D mesh (Mesh-B) used in this study, and thus more LES activity is expected. This is the reason why Gieseking and the modified IDDES models provide more accurate predictions in most cases than the original IDDES model. It can also be noticed that all the hybrid (Gieseking, modified IDDES, and original IDDES) methods predict the separated shear layer above the upper surface and below the bottom surface of the square cylinder, which render a region of lower eddy viscosity on the diagonal extension of the square cylinder locates between the end of the shear layer and the front of the wake area.

\subsection{Effects of spanwise resolution on 3D simulations by modified IDDES model}

The modified IDDES model was selected to perform more 3D simulations (on Mesh-A through Mesh-D) in order to investigate the effects of spanwise resolution. As shown in Table 2, Mesh-A through Mesh-D have the same spanwise extension (of 0.6 D) but different cell spacing, where Mesh-A has the finest resolution and Mesh-D has the coarsest resolution in spanwise direction.

\subsubsection{Vortex shedding frequency, lift and drag forces}

Table 4 shows the aerodynamic characteristics calculated based on the solution of 3D simulations with the modified IDDES model. Strouhal numbers predicted by 3D simulation on Mesh-A, Mesh-B, and Mesh-C are all within the range of experimental measurements. However, 3D simulation on Mesh-D underestimated St with an error up to 21\%. 3D Simulations on Mesh-A and Mesh-B slightly underestimated time-averaged base-pressure coefficient $\left(-\mathrm{C}_{\mathrm{pb}}\right)$ with an error less than $3 \%$, while predictions of time-averaged $-C_{\mathrm{pb}}$ by simulation on Mesh-C and Mesh-D are within the range of experiments. Time averaged drag coefficient $\left(C_{D}\right)$ predicted by 3D simulation increases with the spanwise cell spacing of the $3 \mathrm{D}$ mesh used. $\mathrm{C}_{\mathrm{D}}$ predictions by $3 \mathrm{D}$ simulation on Mesh- $B$, Mesh-C, and Mesh-D are within the range of experiments, while simulation on Mesh-A slightly underestimated $C_{D}$ with an error of $1 \%$. For all the $3 \mathrm{D}$ cases,

Table 4 Results obtained by 3D simulations with modified IDDES model on Mesh-B

\begin{tabular}{lllllll}
\hline Model & Mesh & St & $-C_{p b}$ & $C_{D}$ & $C_{L^{\prime}}$ & $C_{D^{\prime}}$ \\
\hline modified IDDES & 3D Mesh-A & 0.127 & 1.27 & 2.0 & 1.26 & 0.233 \\
modified IDDES & 3D Mesh-B & 0.128 & 1.28 & 2.02 & 1.18 & 0.189 \\
modified IDDES & 3D Mesh-C & 0.126 & 1.32 & 2.06 & 1.31 & 0.314 \\
modified IDDES & 3D Mesh-D & 0.094 & 1.45 & 2.14 & 1.49 & 0.484 \\
Experiments & & $0.122 \sim 0.133$ & $1.3 \sim 1.6$ & $2.02 \sim 2.22$ & $1.18 \sim 1.32$ & $0.207 \sim 0.245$ \\
\hline
\end{tabular}


both $-C_{p b}$ and $C_{D}$ were averaged over 20 vortex shedding periods (with about 3800 time points). The rms $\mathrm{C}_{\mathrm{L}}$ predictions by $3 \mathrm{D}$ simulations on Mesh-A, Mesh-B and Mesh-C are within the range of experiments, while simulation on Mesh-D overestimated the value with an error of 13\%. 3D Simulation on Mesh-A is the only case that provides a prediction on rms $C_{D}$ within the range of experiments. 3D Simulation on Mesh-B underestimates rms $C_{D}$ with an error of 9\%, while 3D Simulations on Mesh-C and Mesh-D overestimate $r m s C_{D}$, with errors of $28 \%$ and $98 \%$, respectively.

\subsubsection{Surface pressure coefficient distribution}

Figure 11 shows the average surface pressure coefficient $\left(\mathrm{C}_{\mathrm{p}}\right)$ and $r m s$ pressure coefficient $\left(\mathrm{C}_{\mathrm{p}}{ }^{\prime}\right)$ distributions obtained by 3D simulations using the modified IDDES model. The average surface pressure coefficients predicted by 3D simulations on Mesh-A, Mesh-B and Mesh-C were slightly overestimated over the top, rear and bottom surfaces of the square cylinder compared with the experiments, with predictions by simulation on Mesh-C matching the experiments the best. The predictions of $r m s$ pressure coefficient by 3D simulations on Mesh-A, Mesh-B are in good agreement with Bearman [2] measurements on the rear surface, while prediction by $3 \mathrm{D}$ simulation on Mesh-C is in good agreement with experimental measurements by Noda et al. [4] and Nishimura and Taniike [3]. The prediction of average surface pressure coefficient obtained by the

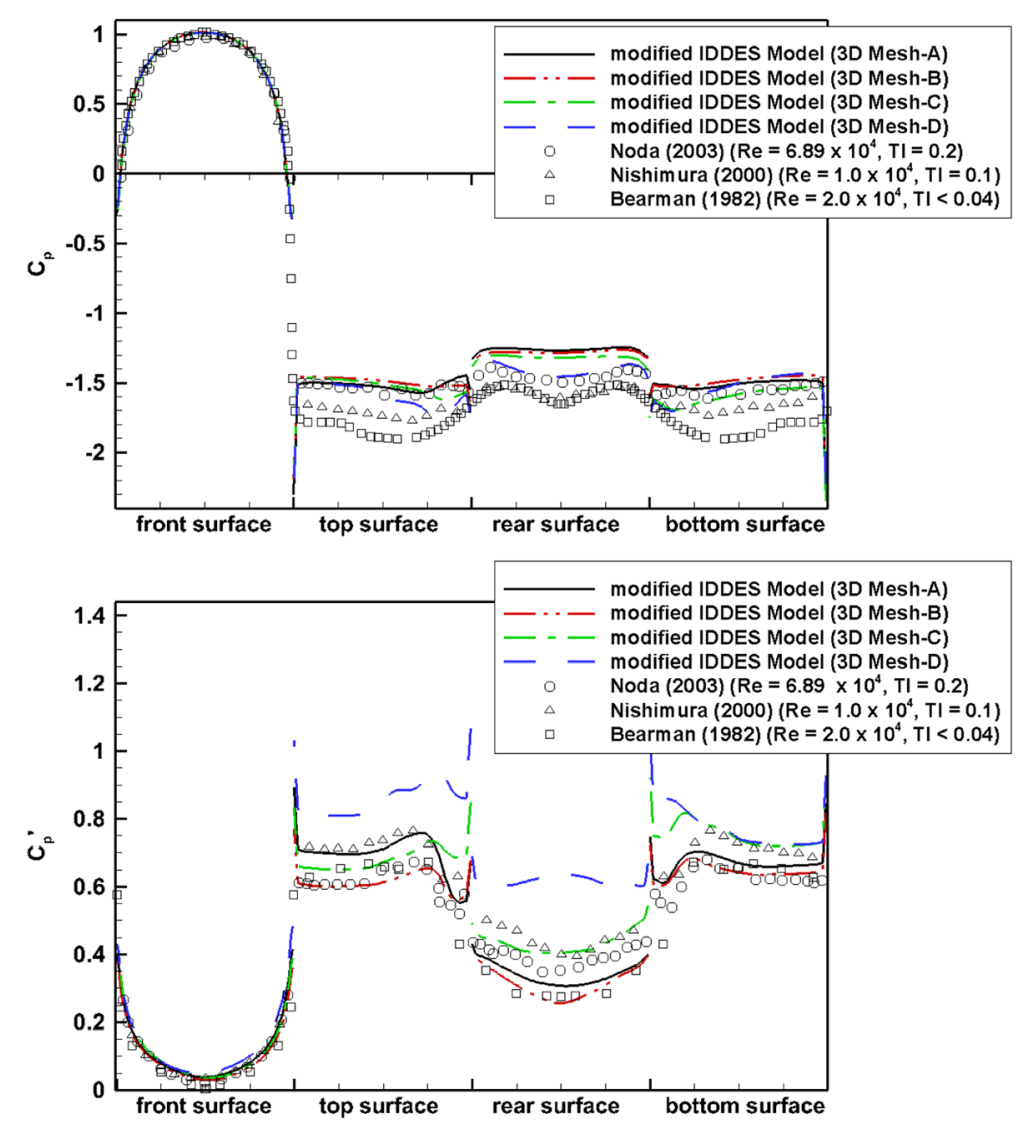

Fig. 11 Surface pressure coefficient distribution (top) and rms pressure coefficient distribution (bottom) by 3D simulations with the modified IDDES model 
IDDES simulation on Mesh-D is also in good agreement with the experiments and even more accurate than Mesh-C on top and rear surfaces of the square cylinder, but the rms pressure coefficient prediction by Mesh-D is inaccurate, especially on the top and rear surfaces.

\subsubsection{Velocity profiles and rms velocity fluctuations}

Figure 12 shows the profiles of mean streamwise and cross-stream velocity components ( $u$ and $v)$ upwind of the wake and near wake $(x / D=0.875)$ predicted by $3 \mathrm{D}$ simulations using the modified IDDES model. The 3D IDDES simulations on Mesh-A, Mesh-B, and Mesh-C accurately predicted the mean streamwise and cross-stream velocity profiles upwind of the wake region, with maximum error generally less than 5\% compared with Lyn's experimental measurements. In the near wake $(x / D=0.875)$, the streamwise

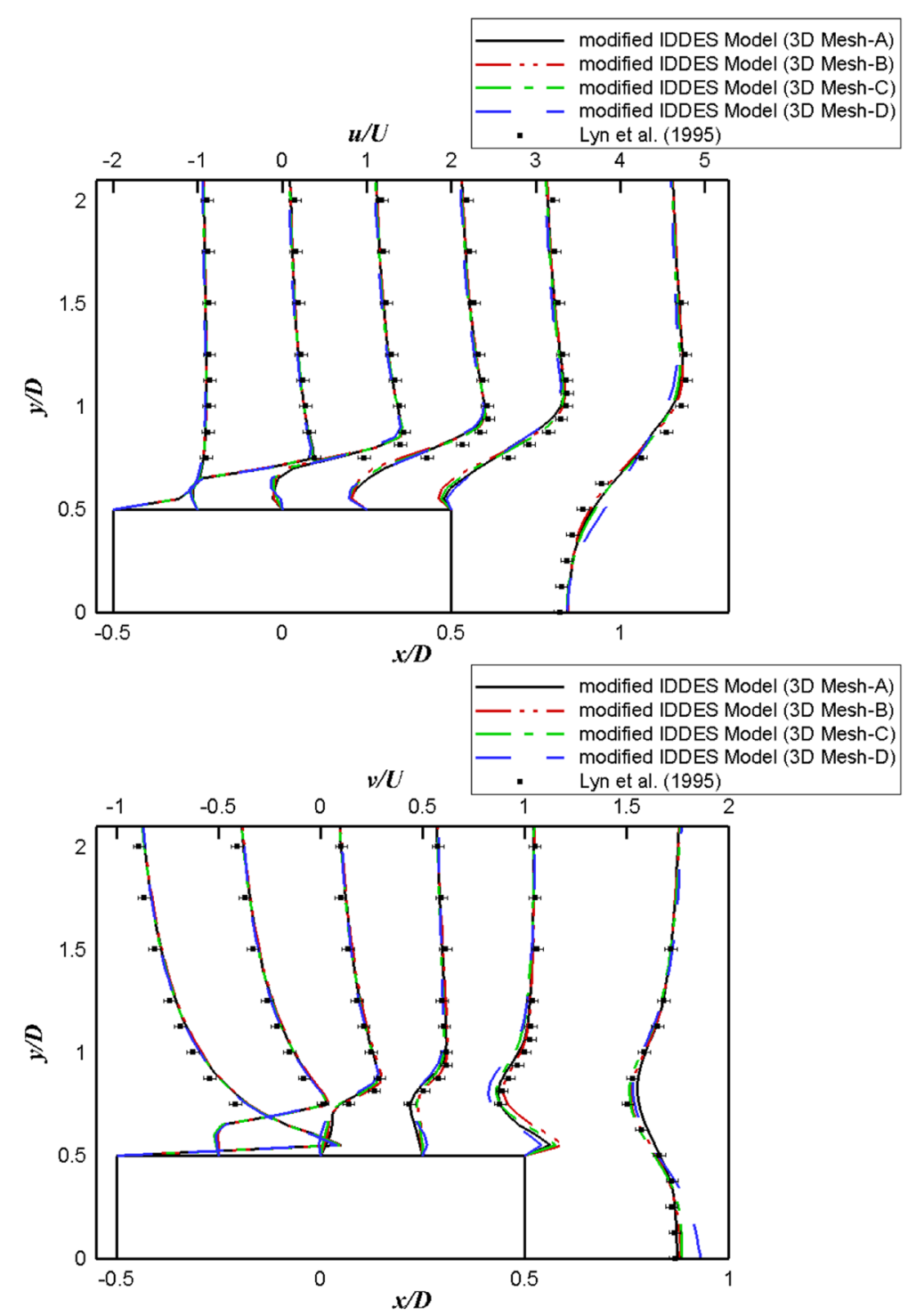

Fig. 12 Mean streamwise (top) and cross-stream (bottom) velocity profiles at different locations near the cylinder predicted by 3D simulations using the modified IDDES model 
and cross-stream velocity profiles obtained by the 3D simulations on Mesh-A, Mesh-B, Mesh- $C$ are also similar to each other with maximum error less than $6 \%$ compared with Lyn's experiments. The predictions of mean $u$ and $v$ by 3D case on Mesh-D are the least accurate against the experiments, especially in the near wake.

As shown in Fig. 13, the predictions of mean streamwise velocity profile along the wake centerline by all 3D simulations are close to Lyn's experiments, among which the 3D case on Mesh-C performs the best against the experiments, especially along the centerline in the near wake and far wake regions. However, the mean streamwise velocity is slightly underestimated by 3D IDDES simulation on Mesh-C along the centerline between locations $x / D=1.5$ and 3.0.

As shown on Fig. 14, the rms streamwise and cross-stream velocity fluctuations obtained by 3D simulation on Mesh-B are in the best agreement with Lyn's experiments in the region far from the wall $(y / D>0.75)$, but are slightly underestimated in the near wake $(x / D=0.875)$, with maximum errors being $20 \%$ and $15 \%$, respectively. The rms streamwise velocity fluctuation obtained by 3D simulations on Mesh-A and Mesh-C are in good agreement with Lyn's experiments in both the upwind of the wake and near wake $(x / D=0.875)$ regions (with maximum error less than $5 \%)$. The rms cross-stream velocity fluctuation obtained by 3D simulations on Mesh-A and Mesh-C are also in good agreement with Lyn's experiments, with maximum errors of $15 \%$ in the upwind-of-the-wake region and $5 \%$ in the near-wake region. The rms streamwise and cross-stream velocity fluctuations obtained by 3D simulation on Mesh-D are the least accurate against Lyn's experiments, especially in the near wake. The Reynolds-averaged shear stress predictions by all 3D simulations are in good agreement with the experimental measurements, as shown in Fig. 15, among which the 3D case on Mesh-B performs the best against the experiments.

Figure 16 shows the profiles of rms streamwise velocity (top) and cross-stream velocity (bottom) fluctuations along the centerline in the wake region obtained by 3D simulations using the modified IDDES model. Among all 3D simulations, the case on Mesh-C provides the best performance in predicting both $r m s$ streamwise velocity fluctuation against the experiments, especially in the far wake regions and rms cross-stream velocity fluctuation against the experiments, especially in the near and far wake region. The rms streamwise velocity fluctuation is slightly overestimated by the $3 \mathrm{D}$ simulation on Mesh-C along the centerline in the region between $x / D=1.0$ and 3.0, while $r m s$ cross-stream velocity fluctuation is slightly underestimated along centerline in the region between $x / D=1.5$ and 3.0, where the 3D case on Mesh-A performs the

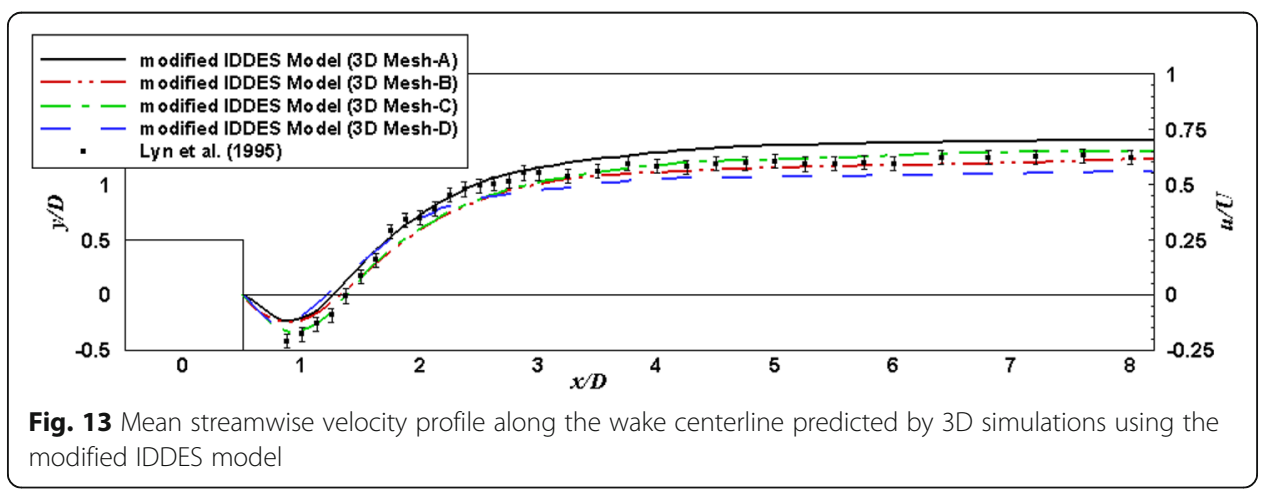




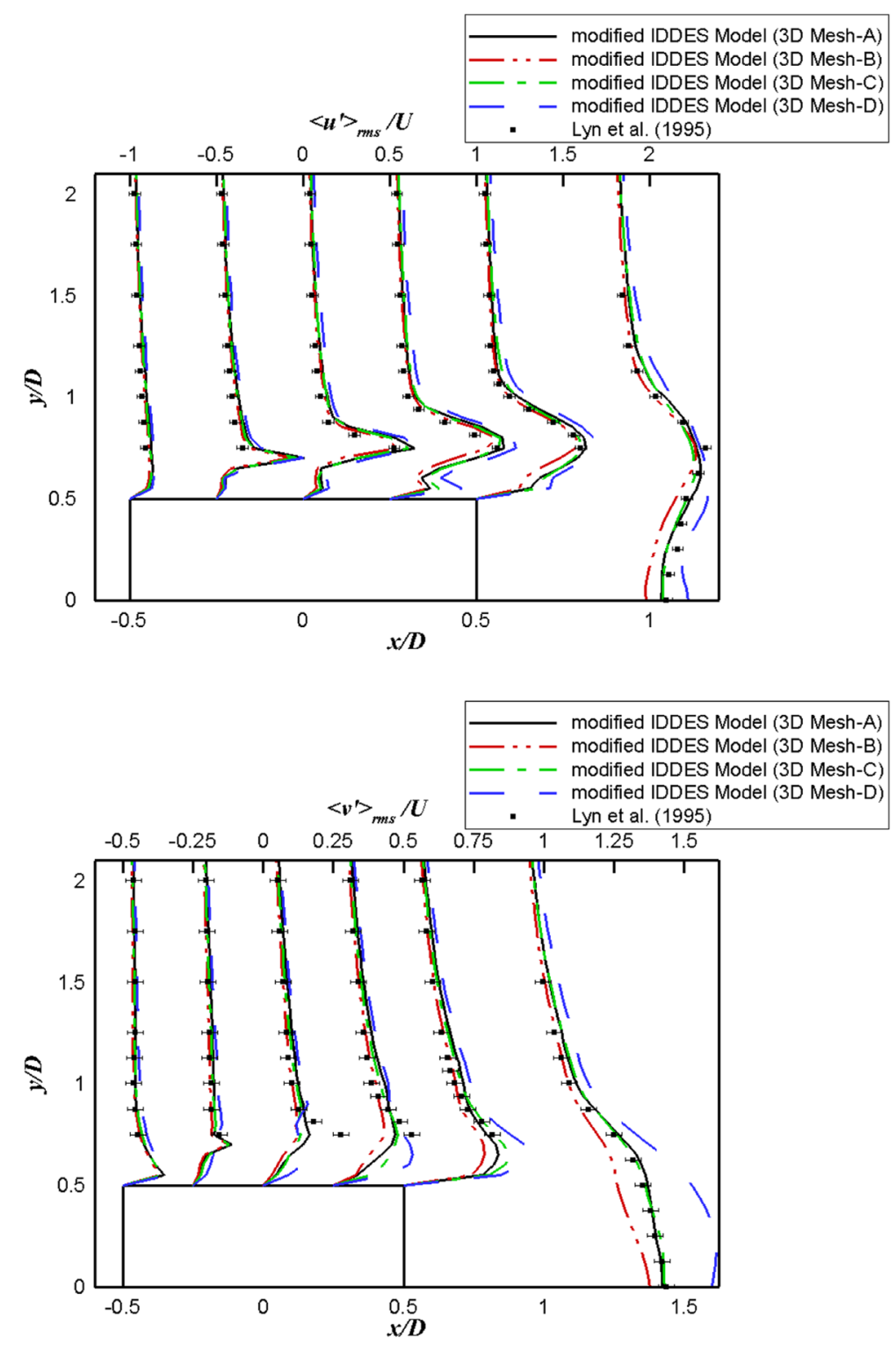

Fig. 14 rms streamwise (top) and cross-stream (bottom) velocity fluctuations at different locations before wake predicted by $3 \mathrm{D}$ simulations with the modified IDDES model

best against the experiments. Again, the 3D simulation on Mesh-D provides the least accurate predictions on rms streamwise and cross-stream velocity fluctuations against the experiments, especially in the near wake region.

Among all 3D simulations using the modified IDDES model, the case on Mesh-D shows the worst performance in predicting most aerodynamic characteristics, rms pressure coefficient, mean $u$ and $v$ velocity profiles as well as second order statistics in both upwind of the wake and near and far wake regions. This is due to the mesh resolution along the spanwise direction being not fine enough to resolve some of the turbulent vortices by LES model. On the other hand, the case on mesh with the finest cell spacing along spanwise direction (Mesh-A) does not always provides the most accurate predictions on some of the aerodynamic characteristics. For example, time-averaged 


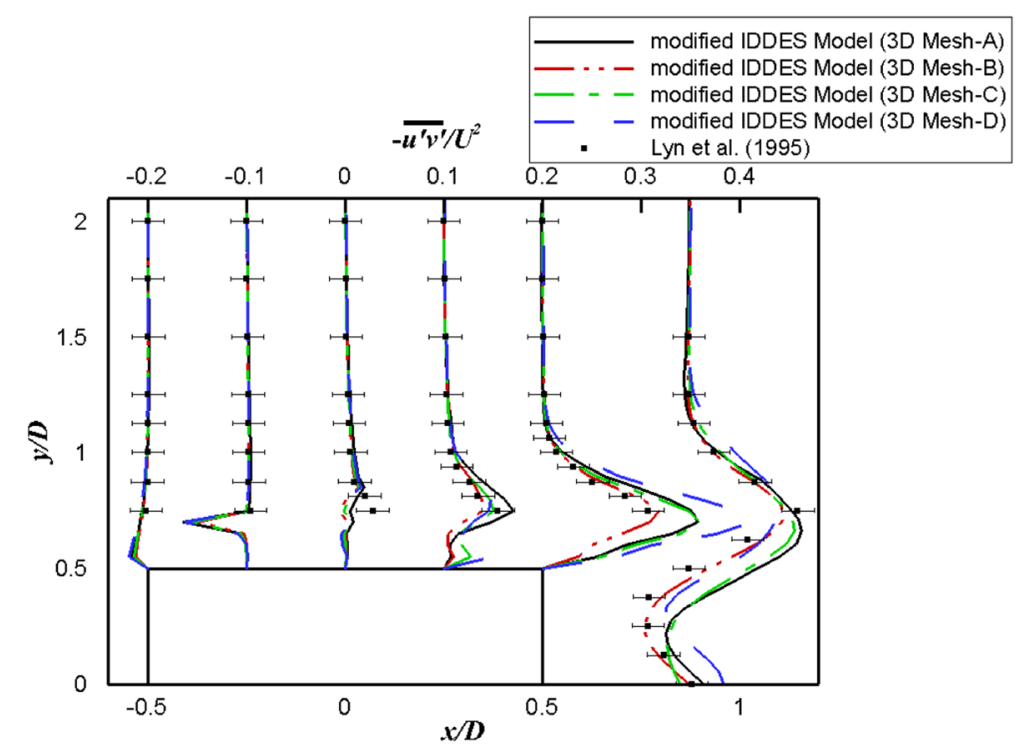

Fig. 15 Reynolds-averaged shear stress profile at different locations before wake predicted by 3D simulations with the modified IDDES model

$-\mathrm{C}_{\mathrm{pb}}$ and $\mathrm{C}_{\mathrm{D}}$ are underestimated by the $3 \mathrm{D}$ simulation on Mesh- $\mathrm{A}$ against the experiments, while the mean streamwise velocity, rms streamwise and cross-stream velocity fluctuations along the wake centerline were overestimated by this case in the far wake region. As a comparison, time-averaged $-C_{p b}$ and $C_{D}$ predicted by the $3 \mathrm{D}$ simulation on Mesh-C (with a coarser spanwise cell spacing than Mesh-A) are in good agreement with the experiments, and the predictions on the mean streamwise velocity, rms streamwise and cross-stream velocity fluctuations along the wake centerline by this case are in better agreement with the experiments. This means that mesh refinement along spanwise direction (say in Mesh-A and Mesh-B) does not guarantee a better 3D

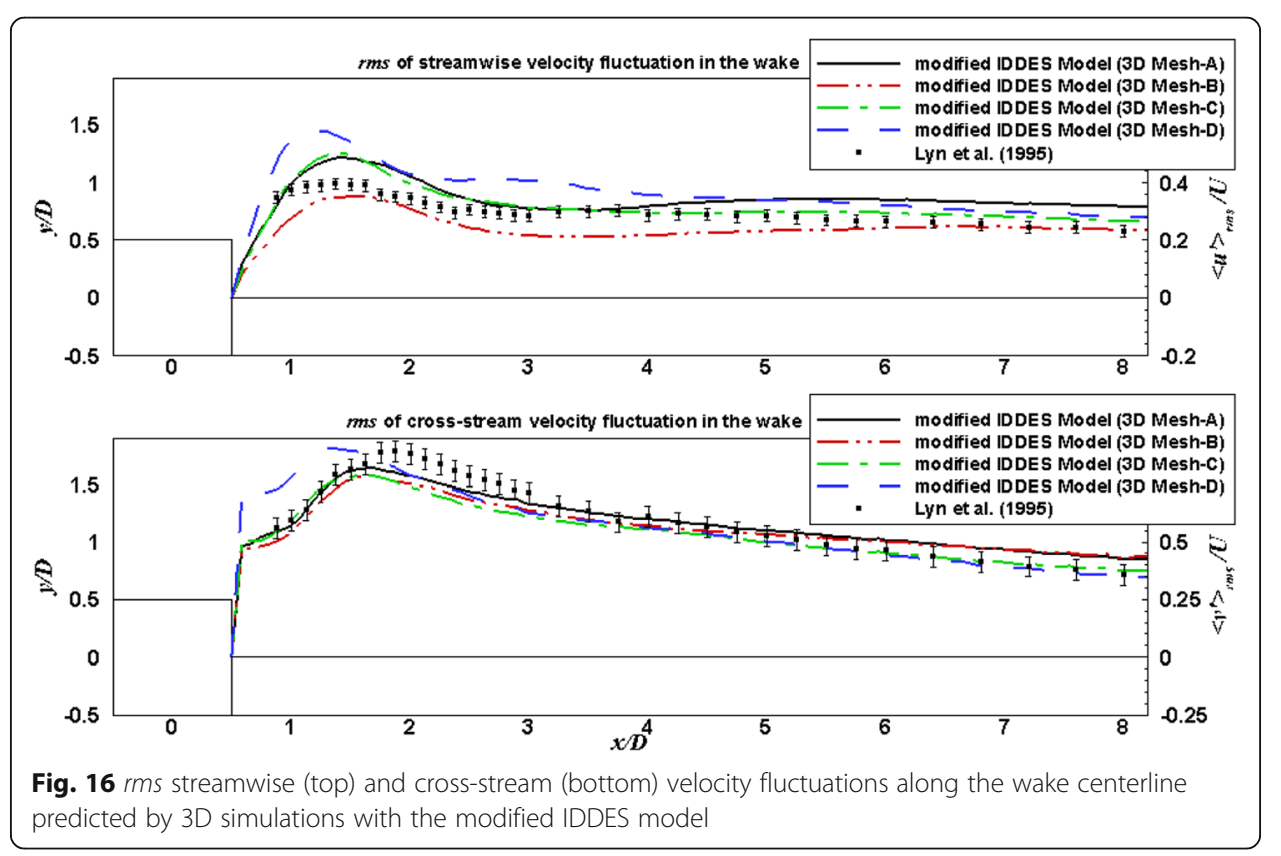


solution with the modified IDDES model. There exists some appropriate spanwise cell spacing for which IDDES model works the best. Similar thing also happens in the 3D hybrid LES/RANS simulations of turbulent flow over NACA 0012 airfoil under static stall [24]. This bifurcation like response appears to be driven by the better resolution of energy-containing three-dimensional eddy structures near the front surface with spanwise mesh refinement. These eddies promote flow attachment and serve to shift the RANS component of the closure closer to the surface. The coarser mesh captures a more two-dimensional response, delaying the transition from RANS to LES and reducing the resolved-eddy energy content.

\section{Conclusions}

Unsteady RANS (URANS) simulations based on Menter's SST model, and 3D simulations based on hybrid LES/RANS and IDDES models were conducted to investigate the turbulent flow over and pressure on a square cylinder at a high Reynolds number. The performance of URANS model in predicting the wake is poor: the rms pressure coefficient on the square cylinder rear surface is underestimated by up to $75 \%$, and the streamwise velocity and $r m s$ streamwise and cross-stream velocity fluctuations obtained by URANS simulations are significantly different from the experiments. Hybrid LES/RANS and IDDES models improve the prediction in the wake region significantly. Compared with the results predicted by URANS simulations, hybrid LES/RANS and IDDES models also provide more accurate predictions against experimental data on most aerodynamic characteristics such as Strouhal number, time averaged base-pressure and drag coefficients, rms lift and drag coefficients, streamwise and cross-stream velocity profiles, as well as rms streamwise and cross-stream velocity fluctuations. The solutions obtained by Gieseking's model and the modified IDDES model on the same 3D mesh are very similar to each other, so do the aerodynamic characteristics predicted by the two models, generally both predicting more accurately on most of the aerodynamic characteristics against experiments than the original IDDES model on the same 3D mesh. However, mesh refinement along spanwise direction does not guarantee a better 3D solution by using the modified IDDES model, there exists some appropriate spanwise cell spacing for which the modified model works the best.

This work was performed while the author served as the National Institute of Standards and Technology (NIST) Director's Postdoctoral Research Associate. The funding comes from the Structure Performance for Multi-hazards Program provided by Materials and Structure Systems Division of Engineering Lab of NIST.
}

\section{Availability of data and materials}

The datasets generated during and/or analysed during the current study are available from the corresponding author on reasonable request.

Authors' contributions

$\mathrm{JK}$ is the only author of this manuscript; he conducted all the work regarding numerical simulations and analysed the numerical results; he also wrote the manuscript. The author read and approved the final manuscript.

Authors' information

Dr. Ke used to be a Postdoctoral Research Associate at National Institute of Standards and Technology (NIST) and this study was done at NIST. Dr. Ke obtained his PHD degree of Aerospace Engineering at North Carolina State University. Currently Dr. Ke is working on aerodynamics in brushless motor as a research assistant at Department of Energy and Power Engineering of Tsinghua University. 


\section{Competing interests}

The author declares that he has no competing interests.

\section{Publisher's Note}

Springer Nature remains neutral with regard to jurisdictional claims in published maps and institutional affiliations.

\section{Received: 17 January 2019 Accepted: 28 March 2019}

Published online: 02 May 2019

\section{References}

1. Kim Y, Kanda J (2010) Characteristics of aerodynamic forces and pressures on square plan buildings with height variation. J Wind Eng Ind Aerodynamics 98(8-9):449-465

2. Bearman P, Obasaju E (1982) An experimental study of pressure fluctuations on fixed and oscillating square-section cylinders. J Fluid Mech 119:297-321

3. Nishimura H, Taniike $Y$ (2000) Fluctuating wind forces on a stationary two-dim square prism. In Proceedings of 16th National Symposium on Wind Engineering in Japan, 255-260

4. Noda H, Nakayama A (2003) Free-stream turbulence effects on the instantaneous pressure and forces on cylinders of rectangular cross section. Exp Fluids 34(3):332-344

5. Lyn D, Einav S, Rodi W, Park J (1995) A laser-Doppler velocimetry study of ensemble-averaged characteristics of the turbulent near wake of a square cylinder. J Fluid Mech 304:285-319

6. Younis BA, Abrishamchi A (2014) Three-dimensional turbulent vortex shedding from a surface-Mounted Square cylinder: predictions with large-Eddy simulations and URANS. J Fluid Eng Trans ASME 136(6):060907

7. Younis BA, Przulj VP (2006) Computation of turbulent vortex shedding. Comput Mech 37:408-425

8. Bouris D, Bergeles G (1999) 2D LES of vortex shedding from a square cylinder. J Wind Eng Ind Aerodynamics 80(1-2):31-46

9. Lakehal D, Thiele F, Lageneste L, Buffat M (1998) Computation of Vortex-Shedding Flows Past a Square Cylinder Employing LES and RANS. In: Hirschel EH (eds) Numerical Flow Simulation I. Notes on Numerical Fluid Mechanics (NNFM), vol 66. Springer, Heidelberg, p 260-277

10. Mahir N (2009) Three-dimensional flow around a square cylinder near a wall. Ocean Eng 36(5):357-367

11. Lim HC, Thomas TG, Castro IP (2009) Flow around a cube in a turbulent boundary layer: LES and experiment. J Wind Eng Ind Aerodynamics 97(2):96-109

12. Srinivas $Y$, Biswas G, Parihar AS, Ranjan R (2006) Large-Eddy simulation of high Reynolds number turbulent flow past a square cylinder. J Eng Mech 132(3):327-335

13. Oka S, Ishihara T (2009) Numerical study of aerodynamic characteristics of a square prism in uniform flow. J Wind Eng Ind Aerodynamics 97(11-12):548-559

14. Bosch G, Rodi W (1998) Simulation of vortex shedding past a square cylinder with different turbulence models. Int J Numer Methods Fluids 28(4):601-616

15. Gieseking D, Choi J, Edwards J, Hassan H (2011) Compressible flow simulations using a new LES/RANS model. AIAA J 49(10): 2194-2209

16. Spalart P, Jou W, Strelets M, Allmaras S (1997) Comments of feasibility of LES for wings, and on a hybrid RANS/LES approach. In Proceedings of First AFOSR International Conference on DNS/LES, Greyden Press, 1997

17. Spalart P, Deck S, Shur M, Squires K, Strelets M, Travin A (2006) A new version of detached-Eddy simulation resistant to ambigous grid densities. Theor Comput Fluid Dyn 20(3):181-195

18. Shur M, Spalart P, Strelets M, Travin A (2008) A hybrid RANS-LES approach with delayed-DES and wall-modelled LES capalities. Int J Heat Fluid Flow 29(6):1638-1649

19. Roy, C., Blottner, F., and Payne, J., 2003. Bluff-body flow simulations using hybrid RANS/LES. $33^{\text {rd }}$ AIAA fluid dynamics conference and exhibit, fluid dynamics and co-located conferences, AIAA 2003-3889

20. Barone M, Roy C (2006) Evaluation of detached Eddy simulation for turbulent wake applications. AIAA J 44(12):3062-3071

21. Morgan, P., and Visbal, M., 2006. Application of hybrid RANS/LES to geometries with separated flows. $3^{\text {rd }}$ AIAA flow control conferences, fluid dynamics and co-located conferences. AIAA 2006-3028

22. Camarri S, Salvetti M, Koobusy B, Dervieu A (2004) An hybrid RANS/LES approach applied to bluff-body flow simulation. In: European congress on computational methods in applied science and engineering, ECCOMAS

23. Ke J (2014) RANS and hybrid LES/RANS simulation of airfoil under static and dynamic stall. PHD Disertation, North Carolina state Uniersity, Raleigh

24. Ke J, Edwards JR (2017) Numerical simulations of turbulent flow over airfoils near and during static stall. J Aircr 54(5):1960-1978

25. Lenormand E, Sagaut P (2000) Subgrid-scale models for large-Eddy simulations of Compressible Wall bounded flows. AIAA J 38(8):1340-1350

26. Menter F (1994) Two-equation eddy-viscosity turbulence models for engineering applications. AIAA J 32(8):1598-1605

27. Colella P, Woodward P (1984) The piecewise parabolic method (PPM) for gas-dynamical simulations. J Comput Phys 54:174-201

28. Cassidy D, Edwards J, Tian M (2009) An investigation of Interface-sharpening schemes for multiphase mixture flows. J Comput Phys 228(16):5628-5649

29. Vickery B (1966) Fluctuating lift and drag on a long cylinder of square cross-sectioin in a smooth and in a turulent stream. J Fluid Mech 25(3):481-494

30. Igarashi $T$ (1984) Characteristics of the flow around a squre prism. Bulletin of JSME 27(231):1858-1865

31. Durao D, Heitor M, Pereira J (1988) Measurements of turbulent and periodic flows around a square cross-section cylinder. Exp Fluids 6(5):298-304

32. Lee $B(1975)$ The effect of turbulence on the surface pressure field of a square prism. J Fluid Mech 69(2):263-282

33. Liu M, Xiao L, Yang L (2015) Experimental investigation of flow characteristics aournd four square-cylinder arrays at subcritical Reynolds numbers. Int J Naval Architecture Ocean Eng 7:906-919

34. Alam M, Zhou Y, Wang X (2011) The wake of two side-by-side square cylinders. J Fluid Mech 669:432-471 